\title{
KOI-54: THE KEPLER DISCOVERY OF TIDALLY EXCITED PULSATIONS AND BRIGHTENINGS IN A HIGHLY ECCENTRIC BINARY*
}

\author{
William F. Welsh ${ }^{1}$, Jerome A. Orosz ${ }^{1}$, Conny Aerts ${ }^{2,3}$, Timothy M. Brown ${ }^{4,5}$, Erik Brugamyer ${ }^{6}$, William D. Cochran ${ }^{6}$, \\ Ronald L. Gilliland ${ }^{7}$, Joyce Ann GuZiK ${ }^{8}$, D. W. Kurtz ${ }^{9}$, David W. Latham ${ }^{10}$, Geoffrey W. Marcy ${ }^{11}$, \\ Samuel N. QuinN ${ }^{10}$, Wolfgang Zima ${ }^{2,3}$, Christopher Allen ${ }^{12}$, Natalie M. Batalha ${ }^{13,14}$, Steve Bryson ${ }^{14}$, \\ Lars A. Buchhave ${ }^{15,16}$, Douglas A. Caldwell ${ }^{14,17}$, Thomas N. Gautier III $^{18}$, STeve B. Howell ${ }^{14}$, K. Kinemuchi ${ }^{14,19}$, \\ Khadeejah A. Ibrahim ${ }^{12}$, Howard Isaacson ${ }^{11}$, Jon M. Jenkins ${ }^{14,17}$, Andrej Prsa ${ }^{20}$, Martin Still ${ }^{14,19}$, Rachel Street ${ }^{4}$, \\ BiLl WOHLER $^{12}$, David G. Koch ${ }^{14}$, AND William J. BorUCKI ${ }^{14}$ \\ ${ }^{1}$ Astronomy Department, San Diego State University, San Diego, CA 92182, USA; wfw@ sciences. sdsu. edu \\ 2 Instituut voor Sterrenkunde, K. U. Leuven, B-3001 Leuven, Belgium \\ ${ }^{3}$ Department of Astrophysics, IMAPP, University of Nijmegen, 6500 GL Nijmegen, The Netherlands \\ ${ }^{4}$ Las Cumbres Observatory Global Telescope, Goleta, CA 93117, USA \\ ${ }^{5}$ Department of Physics, University of California, Santa Barbara, CA 93106, USA \\ ${ }^{6}$ McDonald Observatory and Department of Astronomy, The University of Texas at Austin, Austin, TX 78712, USA \\ ${ }^{7}$ Space Telescope Science Institute, Baltimore, MD 21218, USA \\ ${ }^{8}$ XTD-2, MS T086, Los Alamos National Laboratory, Los Alamos, NM 87545-2345, USA \\ ${ }^{9}$ Jeremiah Horrocks Institute of Astrophysics, University of Central Lancashire, Preston PR1 2HE, UK \\ ${ }^{10}$ Harvard-Smithsonian Center for Astrophysics, Cambridge, MA 02138, USA \\ ${ }^{11}$ Department of Astronomy, University of California, Berkeley, CA 94720, USA \\ 12 Orbital Sciences Corporation/NASA Ames Research Center, Moffett Field, CA 94035, USA \\ ${ }^{13}$ Department of Physics and Astronomy, San Jose State University, San Jose, CA 95192, USA \\ ${ }^{14}$ NASA Ames Research Center M/S 244-30, Moffett Field, CA 94035, USA \\ 15 Niels Bohr Institute, University of Copenhagen, DK-2100 Copenhagen, Denmark \\ ${ }^{16}$ Centre for Star and Planet Formation, Natural History Museum of Denmark, University of Copenhagen, \\ DK-1350 Copenhagen, Denmark \\ 17 SETI Institute, Mountain View, CA 94043, USA \\ 18 Jet Propulsion Laboratory/California Institute of Technology, Pasadena, CA 91109, USA \\ ${ }^{19}$ Bay Area Environmental Research Inst., Inc., Sonoma, CA 95476, USA \\ ${ }^{20}$ Department of Astronomy and Astrophysics, Villanova University, Villanova, PA 19085, USA \\ Received 2010 December 18; accepted 2011 July 23; published 2011 September 28
}

\begin{abstract}
Kepler observations of the star HD 187091 (KIC 8112039, hereafter KOI-54) revealed a remarkable light curve exhibiting sharp periodic brightening events every 41.8 days with a superimposed set of oscillations forming a beating pattern in phase with the brightenings. Spectroscopic observations revealed that this is a binary star with a highly eccentric orbit, $e=0.83$. We are able to match the Kepler light curve and radial velocities with a nearly face-on $(i=5.5)$ binary star model in which the brightening events are caused by tidal distortion and irradiation of nearly identical A stars during their close periastron passage. The two dominant oscillations in the light curve, responsible for the beating pattern, have frequencies that are the 91st and 90th harmonic of the orbital frequency. The power spectrum of the light curve, after removing the binary star brightening component, reveals a large number of pulsations, 30 of which have a signal-to-noise ratio $\gtrsim 7$. Nearly all of these pulsations have frequencies that are either integer multiples of the orbital frequency or are tidally split multiples of the orbital frequency. This pattern of frequencies unambiguously establishes the pulsations as resonances between the dynamic tides at periastron and the free oscillation modes of one or both of the stars. KOI-54 is only the fourth star to show such a phenomenon and is by far the richest in terms of excited modes.
\end{abstract}

Key words: binaries: close - binaries: spectroscopic - stars: individual (KID 8112039, HD 187091, 2MASS J19461553+4356513) - stars: oscillations - stars: variables: general

Online-only material: color figures

\section{INTRODUCTION}

In its search for transiting extrasolar planets, the Kepler Mission obtains high-precision time series photometry of $\sim 156,000$ stars (Koch et al. 2010; Borucki et al. 2010). This very large high-signal-to-noise sample, combined with the unprecedented near-continuous long-timescale coverage, results in a high potential for discovery of astrophysically important stars. KOI-54 is one of those serendipitous discoveries.

\footnotetext{
* Based in part on observations obtained at the W. M. Keck Observatory, which is operated by the University of California and the California Institute of Technology.
}

Cataloged as HD 187091 before being flagged as Kepler Object of Interest 54, this star was classified as a single bright $(V=8.38)$ A star. The object did not seem unusual in any way prior to these Kepler observations and attracted little attention. This is in stark contrast to the astonishing Kepler light curve, which immediately offered a challenging puzzle begging for elucidation-see Figure 1.

In this paper, we present the Kepler observations and a large set of precise radial velocities that led to our binary star model for KOI-54. We investigate the rich set of oscillations that are present and conclude that these are tidally driven pulsations. We also explore the evolutionary status of these stars and find 

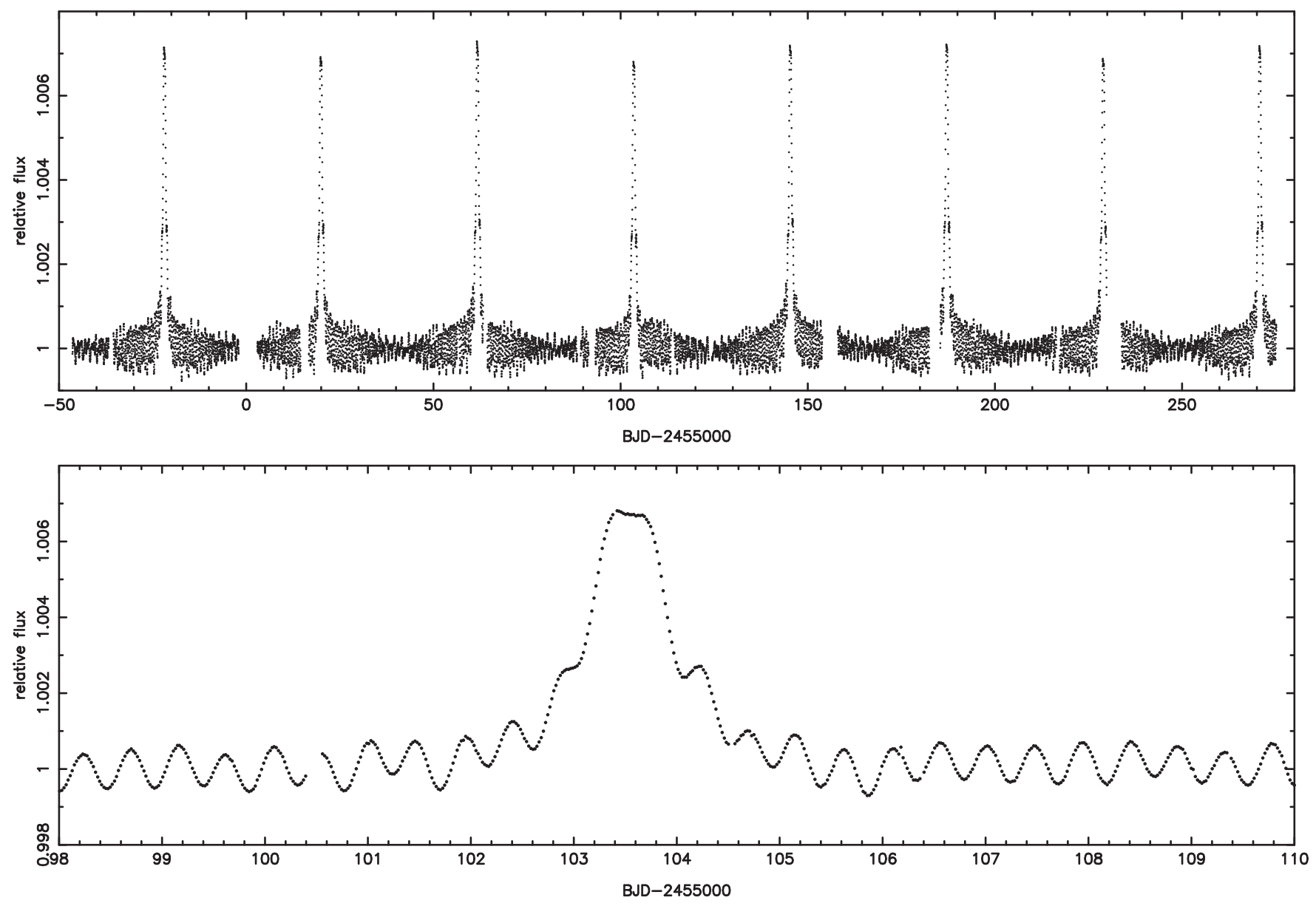

Figure 1. Top: the detrended and normalized Kepler light curve of KOI-54. Bottom: a detailed view of a brightening event.

a self-consistent scenario for the binary system. We finish with some remarks on the puzzles that still remain and discuss in some detail the systematic issues that could potentially affect our interpretation, but we find these issues to generally have no significant effect on our conclusions.

\section{OBSERVATIONS}

\subsection{Overview of the Light Curve}

KOI-54 has been observed by Kepler almost continuously from 2009 May 2 during commissioning observations (Q0) though 2010 March 22 "Quarter 4" (Q4). The light curve exhibits two remarkable features: a periodic brightening spike of $\sim 0.7 \%$ occurring every 42 days and a $\sim 0.1 \%$ "beat pattern" of pulsations in phase with the brightening events. The beat pattern arises from the interference of two pulsations with periods near $11 \mathrm{hr}$. The pulsations continue during the brightening and repeat from event to event-they are strictly in phase with the brightening.

An initial hypothesis that the repeating brightening events might follow from near-field microlensing (Sahu \& Gilliland 2003) was pursued. Such a large event would result from a black hole of several solar masses orbiting and transiting an A star. Motivated by this hypothesis, a 1958 s duration Swift X-Ray Telescope (XRT) observation was made on 2010 April 25 , during one of the brightening events. The source was not detected in the $0.3-10 \mathrm{keV}$ bandpass, but we estimate the $5 \sigma$ upper limit on the X-ray flux to be $F<1.23 \times$ $10^{-13} \mathrm{erg} \mathrm{cm}^{-2} \mathrm{~s}^{-1}$. Using the Hipparcos parallax of 3.14 mas, the upper limit to the luminosity is $\mathrm{L}<1.5 \times 10^{30} \mathrm{erg} \mathrm{s}^{-1}$. Given the null detection, we make no further discussion of these observations.

Radial velocity (RV) observations quickly ruled out the transiting black hole hypothesis because no large rapidly changing reflex motion of the A star was observed. Also, no ready explanation of the oscillations existed in this scenario, and when examined in detail the 42 day brightenings deviate from the expected shape of microlensing events. Instead, as we show below, KOI-54 is a highly eccentric binary star system, and the brightening events are caused by tidal distortion and irradiation of the two stars during their close periastron passage. The two dominant pulsations producing the beating seen in the light curve have frequencies that are exactly 90 and 91 times the orbital frequency, and hence result from tidally driven pulsation modes.

\subsection{The Kepler Photometry and Calibration}

The Kepler CCDs are read out every 6.54 s (6.02 s live time) and co-added on board. In Short Cadence (SC) mode the signal is combined to achieve approximately $58.8 \mathrm{~s}$ sampling cadence, and in Long Cadence (LC) mode the data are binned to 29.424 minute cadence. KOI-54 is heavily saturated in these $6 \mathrm{~s}$ exposures, and blooming affects approximately 30 pixels of the 74 pixel aperture (approximately 7 pixels in diameter plus two columns of 25 pixels). However, the electrons are not lost, and because of Kepler's stability, superb relative photometry is achievable-see Gilliland et al. (2010) for a discussion. For more details on the design and performance of the Kepler 
photometer see Koch et al. (2010) and Jenkins et al. (2010a, 2010b).

Each Kepler pixel spans $\sim 4^{\prime \prime}$ so the very large photometric aperture includes a few very faint (by comparison) background stars. The brightest star (KIC 8112007) has Kp $=17.6(4850$ times fainter than KOI-54), and ground-based images with $1^{\prime \prime}$ seeing show no star brighter than the 18th magnitude within $5^{\prime \prime}$. We conclude that background starlight contaminates the light curve by less than $250 \mathrm{ppm}(0.025 \%)$, and thus variations in the light curve are intrinsic to KOI-54.

In addition to gaps due to the scheduled quarterly spacecraft rolls, safe-mode events and other spacecraft anomalies are present; cosmic rays and other noise sources also contaminate the signal. A simple automated sigma-threshold rejection method could not be used because of the presence of the complex oscillation signal, so the light curve was carefully examined and obvious outliers were omitted by hand: 158 points were removed, leaving a total of 14,277 observations. This corresponds to a duty cycle of an astounding $92.5 \%$ over the span of 10.6 months.

The observations span 321.7 days, nearly eight complete 41.8 day orbital cycles. For each spacecraft roll, the target is on a different CCD with pixels of different sensitivity, and thus jumps in the light curve occur from quarter to quarter. Mean fluxes over the four quarters span $(1.01-1.07) \times 10^{10} e^{-} /$cadence. To correct for these changes, and the more troublesome safe-moderelated, pointing-adjustment, and other medium-timescale discontinuities, the "RAW" pipeline-processed light curves ${ }^{21}$ were detrended in the following way. First, the brightening events were masked in orbital phase from 0.9 to 1.1 (i.e., $20 \%$ of the light curve). Then using gaps in the time series to define sections, the sections were fit with a low-order polynomial (typically cubic, though linear or a constant were used if appropriate). The polynomial was then subtracted from the time series, and the remainder divided by the mean of the polynomial. A value of +1.00 was then added to this quotient giving a relative flux with a mean of unity. The pipeline uncertainty estimates were boosted by a factor of six, based on the rms scatter of the residuals of an early model fit. This scaling factor was chosen for simplicity, though each quarter should have its own scaling (e.g., values of 5.0, 5.5, and 7.3 for three different quarters were found). The scatter in the residuals was significantly in excess of what was expected just from the error bars, but in hindsight this was due to using too few sinusoidal components in our initial modeling (see below). Slightly overestimating the uncertainties has no adverse affect on the estimation of the stellar and orbital parameters, and helps account for systematic noise (e.g., photometric trends after a safe mode or other anomaly) that is not included in the statistical uncertainties.

One month of SC data was obtained during Quarter 3; for the characteristics of Kepler SC data, see Gilliland et al. (2010). These observations contain 43,990 points and span 30.035 days. Examination of these SC data, and their power spectrum at higher frequencies, did not reveal any features not already well resolved in the LC data; therefore only the LC data are used for our analyses.

Finally, the relative timing precision is good to $0.5 \mathrm{~s}$, but the absolute timing is uncertain by as much as $6.5 \mathrm{~s}$, and this systematic uncertainty should be added to the statistical uncertainty in the epoch of periastron $T_{p}$ in Table 2-see Kepler Data Release 8 Notes (Machalek \& Christiansen 2010).

\footnotetext{
21 Available at the Multimission Archive (MAST) at the Space Telescope Science Institute: http://archive.stsci.edu/kepler/data_search/search.php.
}

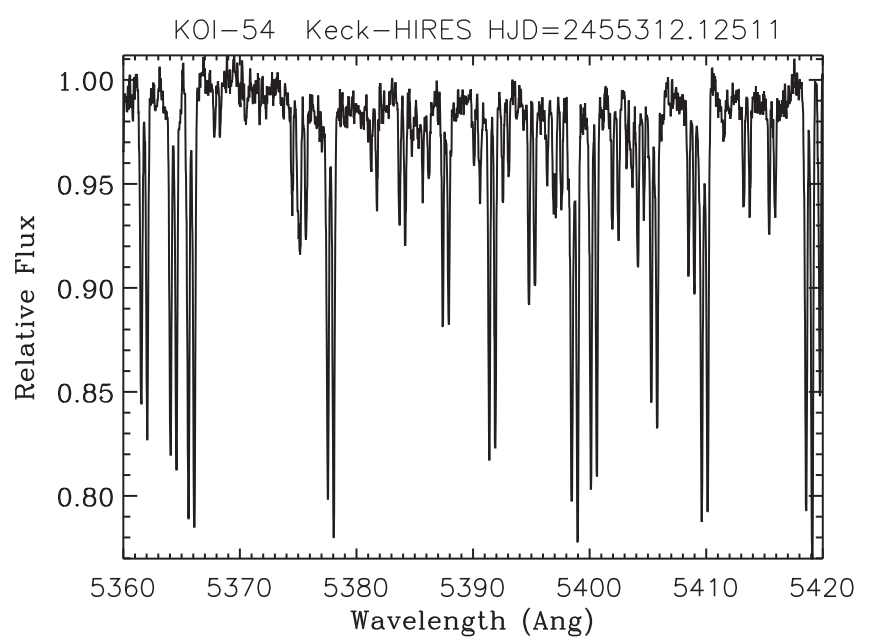

Figure 2. Keck HIRES spectrum showing the double-line absorption and revealing the binary nature of KOI-54.

\subsection{Spectroscopy and Radial Velocities}

A variety of telescopes and instruments were used to provide moderate to high resolution spectroscopy for $\mathrm{RV}$ and spectral modeling. The spectra show double sets of absorption lines revealing the binary star nature of the system, and in Figure 2 we show a section of a Keck HIgh Resolution Echelle Spectrometer (HIRES) spectrum where pairs of lines are clearly exhibited. The stars are of similar spectral type and luminosity. To determine the stellar parameters we carried out an analysis of 11 relatively clean Fe I and Fe II lines using the LTE spectral synthesis code MOOG (Sneden 1973), modified for binary star analysis. Using an input line list and two separate model atmospheres, the code computes a synthetic spectrum for each stellar component. Given a velocity separation and luminosity ratio, the code then overplots the resulting spectra onto the observed spectrum. We then varied the stellar parameters (temperature, gravity, metallicity, and microturbulent velocity) until the best overall match to our selected iron lines was found. Using a Hobby-Eberly Telescope (HET) High Resolution Spectrograph (HRS) spectrum taken very near periastron (when the stars were cleanly separated in Doppler velocity by $25.5 \mathrm{~km} \mathrm{~s}^{-1}$ ), we obtained the following information about the stars. The stellar temperatures $T_{1}$ and $T_{2}$ are 8500 and $8800 \mathrm{~K} \pm 200 \mathrm{~K}$, with $\log g$ of 3.8 and $4.1 \pm 0.2$, and a luminosity ratio $L_{2} / L_{1}$ of $1.22 \pm 0.04$ for the wavelength range covered in the spectral analysis, 4500-6500 $\AA$. Both stars are 2-3 times more metal-rich than solar with $[\mathrm{Fe} / \mathrm{H}]=0.4 \pm 0.2$. Thus the stars are both A-type near-main-sequence stars and potentially pulsating $\delta$ Scuti variables - see Aerts et al. (2010) for a thorough discussion of $\delta$ Scuti and other pulsating stars.

Estimates of $V_{\text {rot }} \sin i$ depended on the spectra and method used, but all indicate a low projected velocity ranging from $\lesssim 5$ to $10 \mathrm{~km} \mathrm{~s}^{-1}$. Given the difficulty in measuring $V_{\text {rot }} \sin i$ we adopt a value of $7.5 \pm 4.5 \mathrm{~km} \mathrm{~s}^{-1}$. When corrected for the very low inclination of the system (discussed in Section 3 ) the true $V_{\text {rot }}$ can be $\lesssim 50-100 \mathrm{~km} \mathrm{~s}^{-1}$, and this is still relatively low for an A-star. This low rotation rate, combined with the metal-rich abundance, suggests that these are chemically peculiar Am or possibly Ap stars. An inspection of the Nd III $6145 \AA$ and Pr III $6160 \AA$ lines show that they are not strong, as they usually are in roAp stars.

A total of 51 pairs of radial velocities were obtained in 2010 from six different telescopes+spectrographs, and these are 
Table 1

KOI-54 Radial Velocities

\begin{tabular}{|c|c|c|c|c|}
\hline HJD-2400000 & $\mathrm{RV}_{1}$ & $\mathrm{RV}_{2}$ & Exp. Time & Facility \\
\hline 55308.9298 & -10.58 & -19.61 & 1080 & TRES \\
\hline 55310.6587 & -8.07 & -21.30 & 900 & FIES \\
\hline 55310.9353 & -7.51 & -22.83 & 360 & TRES \\
\hline 55311.8277 & -3.54 & -26.81 & 577 & MCD \\
\hline 55311.8353 & -3.38 & -26.77 & 547 & MCD \\
\hline 55311.8427 & -3.33 & -26.82 & 529 & $\mathrm{MCD}$ \\
\hline 55311.8775 & -3.10 & -27.24 & 1800 & TRES \\
\hline 55311.9883 & -2.07 & -28.01 & 1800 & TRES \\
\hline 55312.1251 & -1.02 & -28.79 & 72 & HIRES \\
\hline 55312.1265 & -1.03 & -28.93 & 77 & HIRES \\
\hline 55312.1278 & -1.13 & -28.90 & 70 & HIRES \\
\hline 55312.1292 & -0.99 & -28.85 & 72 & HIRES \\
\hline 55312.8216 & -7.07 & -24.23 & 553 & MCD \\
\hline 55312.8300 & -7.10 & -23.91 & 672 & MCD \\
\hline 55312.8899 & -8.37 & -22.69 & 1800 & TRES \\
\hline 55312.9897 & -9.42 & -20.12 & 119 & HIRES \\
\hline 55313.9043 & -16.45 & -13.73 & 1800 & TRES \\
\hline 55313.9888 & -17.13 & -13.47 & 89 & HIRES \\
\hline 55314.8513 & -17.92 & -12.81 & 543 & MCD \\
\hline 55314.9021 & -18.43 & -12.43 & 900 & TRES \\
\hline 55315.0767 & -18.24 & -12.32 & 272 & HIRES \\
\hline 55315.9130 & -18.75 & -12.32 & 1200 & TRES \\
\hline 55316.8788 & -19.12 & -11.90 & 900 & TRES \\
\hline 55317.8700 & -18.23 & -12.06 & 900 & TRES \\
\hline 55318.9274 & -18.45 & -11.91 & 1500 & TRES \\
\hline 55319.0179 & -18.32 & -12.25 & 144 & HIRES \\
\hline 55319.9909 & -18.47 & -12.04 & 1500 & TRES \\
\hline 55320.9937 & -18.44 & -12.26 & 840 & TRES \\
\hline 55321.0389 & -18.00 & -12.60 & 81 & HIRES \\
\hline 55321.9376 & -18.42 & -12.23 & 1680 & TRES \\
\hline 55342.9718 & -14.41 & -16.09 & 1260 & TRES \\
\hline 55343.9095 & -14.03 & -16.30 & 1800 & TRES \\
\hline 55344.8253 & -13.87 & -16.45 & 1260 & TRES \\
\hline 55345.8418 & -13.55 & -17.12 & 1260 & TRES \\
\hline 55346.7917 & -13.32 & -16.99 & 1260 & TRES \\
\hline 55347.8754 & -12.61 & -17.79 & 1800 & TRES \\
\hline 55348.7957 & -12.73 & -17.50 & 1800 & TRES \\
\hline 55349.8360 & -11.90 & -18.97 & 1260 & TRES \\
\hline 55352.7859 & -7.26 & -22.86 & 900 & HET \\
\hline 55352.8244 & -7.07 & -22.86 & 900 & LICK \\
\hline 55352.9850 & -6.72 & -23.70 & 900 & LICK \\
\hline 55353.7790 & -2.21 & -27.67 & 900 & HET \\
\hline 55353.7974 & -1.92 & -27.52 & 900 & LICK \\
\hline 55353.9845 & -0.90 & -29.34 & 900 & LICK \\
\hline 55366.9826 & -17.77 & -12.78 & 1200 & TRES \\
\hline 55367.8032 & -17.85 & -12.72 & 1800 & TRES \\
\hline 55368.7601 & -17.46 & -13.05 & 1800 & TRES \\
\hline 55369.7711 & -17.29 & -13.36 & 1800 & TRES \\
\hline 55370.7447 & -17.40 & -12.99 & 1800 & TRES \\
\hline 55373.7590 & -17.42 & -13.17 & 1260 & TRES \\
\hline 55376.9083 & -16.44 & -14.22 & 1800 & TRES \\
\hline
\end{tabular}

Notes. Velocities reported in units of $\mathrm{km} \mathrm{s}^{-1}$, and exposure times in seconds. Uncertainties are estimated to be $0.31 \mathrm{~km} \mathrm{~s}^{-1}$.

a Facility Code: TRES: Tillinghast Reflection Echelle Spectrograph on the F. L. Whipple Observatory $1.5 \mathrm{~m}$ telescope. FIES: FIbre-fed Echelle Spectrograph on the Nordic Optical Telescope. MCD: Tull spectrograph on the McDonald Observatory $2.7 \mathrm{~m}$ Harlan J. Smith Telescope. HIRES: HIRES spectrograph on the W.M. Keck Observatory Keck I Telescope. LICK: Hamilton Echelle Spectrograph on the Lick Observatory Shane $3 \mathrm{~m}$ Telescope. HET: HRS spectrograph on the McDonald Observatory Hobby-Eberly Telescope.

listed in Table 1. To minimize any potential systematic offset between the velocities acquired with different instruments, all
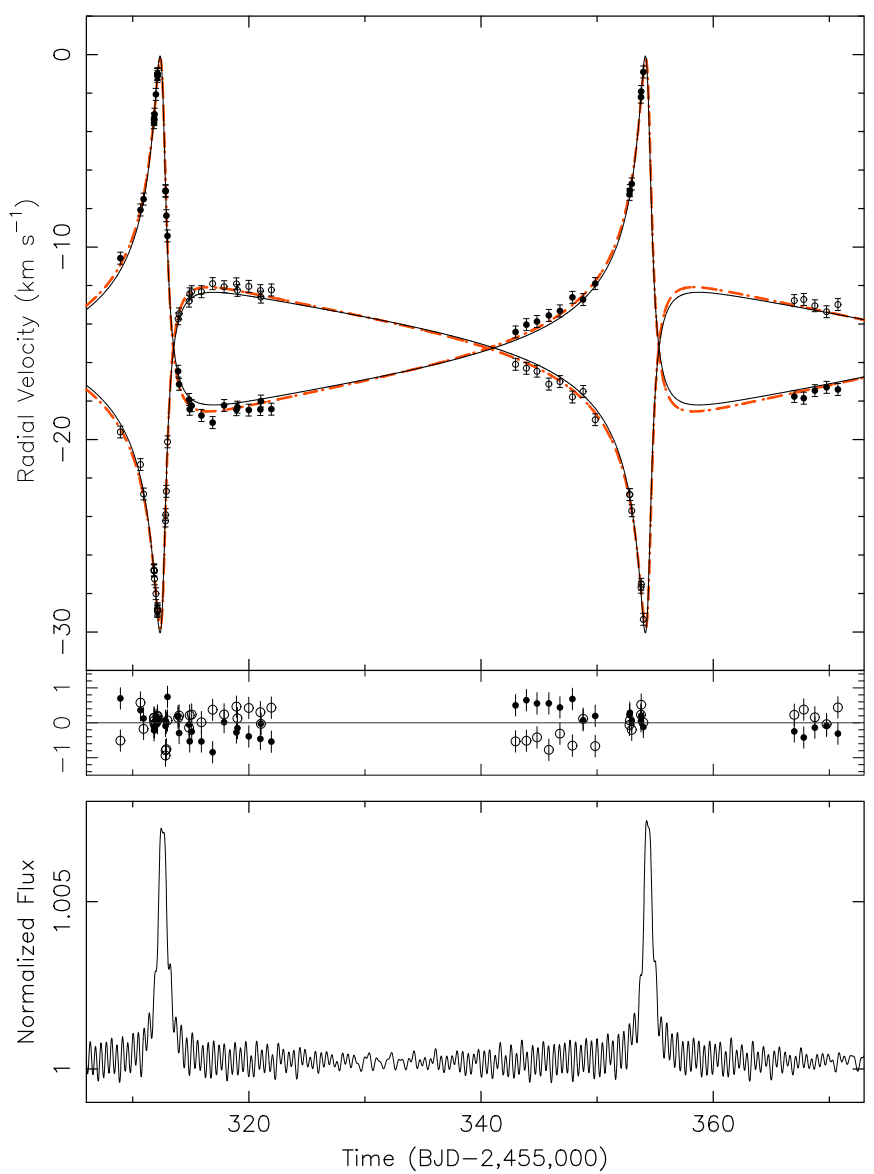

Figure 3. Top: observed radial velocities (RVs) and fits: the dashed line is the fit to the RV data only, the solid line is the best-fit model that simultaneously fits the RV and the light curve. The filled circles denote the RV curve of Star 1. Middle: the residuals of the data minus the best fit. Bottom: the ELCsinus model light curve showing the predicted fluxes at the time of the RV observations.

(A color version of this figure is available in the online journal.)

RV observations were calibrated using the same procedure and all velocities measured using the TODCOR technique (Zucker \& Mazeh 1994). Every velocity measurement is referenced to a common RV standard, HD 182488, that was observed every night, following standard practice for Kepler follow-up observations of targets of interest. Although not simultaneous with the Kepler photometry presented in this paper, these observations provided the key to understanding the nature of KOI-54: the stars are on a highly eccentric orbit, $e=0.83$, with periastron passage at the times of the brightenings. Thus, the mutual interaction of the stars when closest together produces the brightening events seen in the Kepler photometry, and this is discussed in detail in Sections 3 and 5.1. Figure 3 shows the radial velocities and Keplerian fits, and Table 2 includes the orbital elements from the RV-only fit. The ratio of $\mathrm{K}$-velocities gives a mass ratio of 1.034, again confirming the similarity of the two stars. Note: the TODCOR methodology we employed for measuring the radial velocities does not provide uncertainty estimates on the radial velocities, so the mean of the residuals from the RV fit to the observations was assigned as the uncertainty to all the velocities $-0.31 \mathrm{~km} \mathrm{~s}^{-1}$. Given the relatively good fit to the RV data over most of the orbit, this approximation is justifiable. Concerns over systematic errors, as seen in the residuals of the fit, are discussed in Section 5.1.3. 
Table 2

KOI-54 System Parameters

\begin{tabular}{|c|c|c|c|}
\hline Parameter & Value & Uncertainty & Unit \\
\hline Star 1 temperature: $T_{1}$ & 8500 & 200 & $\mathrm{~K}$ \\
\hline Star 2 temperature: $T_{2}$ & 8800 & 200 & $\mathrm{~K}$ \\
\hline $\log g_{1}$ & 3.8 & 0.2 & (cgs) \\
\hline $\log g_{2}$ & 4.1 & 0.2 & (cgs) \\
\hline Luminosity ratio: L2/L1 & 1.22 & 0.04 & \\
\hline Star $1 V_{\text {rot }} \sin i_{1}$ & 7.5 & 4.5 & $\mathrm{~km} \mathrm{~s}^{-1}$ \\
\hline $\operatorname{Star} 2 V_{\text {rot }} \sin i_{2}$ & 7.5 & 4.5 & $\mathrm{~km} \mathrm{~s}^{-1}$ \\
\hline Star $1[\mathrm{Fe} / \mathrm{H}]_{1}$ & 0.4 & 0.2 & \\
\hline $\operatorname{Star} 2[\mathrm{Fe} / \mathrm{H}]_{2}$ & 0.4 & 0.2 & \\
\hline \multicolumn{4}{|l|}{ Fitting RV only: } \\
\hline$K_{1}$ & 9.16 & 0.10 & $\mathrm{~km} \mathrm{~s}^{-1}$ \\
\hline$K_{2}$ & 8.85 & 0.10 & $\mathrm{~km} \mathrm{~s}^{-1}$ \\
\hline Mass ratio, $q=M_{2} / M_{1}$ & 1.034 & 0.016 & \\
\hline Systemic velocity, $\gamma$ & -15.257 & 0.035 & $\mathrm{~km} \mathrm{~s}^{-1}$ \\
\hline Orbital period, $P$ & 41.805 & 0.014 & days \\
\hline Epoch of Periastron, $T_{p}$ & 2455103.5973 & 0.0074 & BJD \\
\hline Orbital eccentricity, $e$ & 0.8315 & 0.0032 & \\
\hline Arg. periastron, $\omega$ & 39.46 & 0.51 & deg \\
\hline \multicolumn{4}{|l|}{ Fitting RV + light curve: } \\
\hline$K_{1}$ & 9.04 & 0.07 & $\mathrm{~km} \mathrm{~s}^{-1}$ \\
\hline$K_{2}$ & 8.82 & 0.09 & $\mathrm{~km} \mathrm{~s}^{-1}$ \\
\hline Mass ratio, $q=M_{2} / M_{1}$ & 1.024 & 0.013 & \\
\hline Systemic velocity, $\gamma$ & -15.239 & 0.034 & $\mathrm{~km} \mathrm{~s}^{-1}$ \\
\hline Orbital period, $P$ & 41.8050 & 0.0003 & days \\
\hline Epoch of periastron, $T_{p}$ & 2455103.5490 & 0.0010 & $\mathrm{BJD}$ \\
\hline Orbital eccentricity, $e$ & 0.8335 & 0.0005 & \\
\hline Arg. periastron, $\omega$ & 36.70 & 0.90 & deg \\
\hline Orbital inclination, $i$ & 5.50 & 0.10 & deg \\
\hline Semimajor axis, $a$ & 0.3956 & 0.008 & $\mathrm{AU}$ \\
\hline Star 1: $\Omega_{1}$ & 3.5 & 2.3 & $\cdots$ \\
\hline Star 2: $\Omega_{2}$ & 1.0 & 0.9 & $\cdots$ \\
\hline Star 1 mass: $M_{1}$ & 2.33 & 0.10 & $M_{\odot}$ \\
\hline Star 2 mass: $M_{2}$ & 2.39 & 0.12 & $M_{\odot}$ \\
\hline Star 1 radius: $R_{1}$ & 2.20 & 0.03 & $R_{\odot}$ \\
\hline Star 2 radius: $R_{2}$ & 2.33 & 0.03 & $R_{\odot}$ \\
\hline
\end{tabular}

Notes. $\Omega$ is defined as the ratio of rotation frequency to the pseudosynchronous rotation frequency: $\Omega \equiv \Omega_{\text {rot }} / \Omega_{\mathrm{ps}}$.

\section{MODELING}

We employ a modified version of the ELC modeling code of Orosz (Orosz \& Hauschildt 2000) to simultaneously fit both the photometry and radial velocities. The non-spherical stars are covered with a fine grid of tiles, and for each time the intensity and velocity of the tiles are summed to produce the light curve and radial velocities. Gravitational distortions are modeled assuming a standard Roche potential, including the rotation of the stars themselves; the potentials are recomputed at each orbital phase because of the elliptical orbit-see the appendix of Orosz \& Hauschildt (2000) for a description of the potential that is based on Avni \& Bahcall (1975) and see Sepinksy et al. (2007) for a thorough discussion of equipotential surfaces in nonsynchronous eccentric binaries. Gravity darkening is included, using an exponent $\beta=0.25$ appropriate for early-type radiative stars (von Zeipel 1924; Claret 2000). We used tabulated spherical NeXTGEN/PHOENIX model intensities (Hauschildt et al. 1997). The model flux is then integrated over the Kepler spectral response (approximately 4250-8950 A, peaking at $5890 \AA$ with a mean wavelength of $6400 \AA$ - see Van Cleve $\&$ Caldwell 2009 and Koch et al. 2010). Irradiation of the stars is handled following the standard prescription of Wilson
(1990). The light from each star is the sum of (1) the intrinsic intensities of each tile (modified for the local gravity and, if blackbodies are used, the limb darkening) and (2) an irradiation "reflection" component on the inward-facing hemispheres. The irradiation modifies the local temperature in the following way: $T^{\prime 4}=T^{4} \times\left[1+A_{\text {bol }} \frac{F_{\text {irr }}}{F}\right]$ where $T$ and $F$ are the temperature and bolometric flux of the star, and $F_{\text {irr }}$ is the incident bolometric flux from the companion star. $A_{\text {bol }}$ is the bolometric albedo (not to be confused with the Bond albedo) and is the ratio of re-radiated-to-incident energy. A radiative atmosphere has $A_{\text {bol }}=1$ (implying local energy conservation), and we hold $A_{\text {bol }}=1$ in our models. Kallrath \& Milone (1999) give an excellent description of Wilson's method and we refer the reader to that source for more details. At periastron, the maximum change in temperature over the surface of the stars, including gravity darkening and irradiation, is $88 \mathrm{~K}(=1 \%)$ for Star 1 and $61 \mathrm{~K}(0.7 \%)$ for Star 2 . At apastron, the difference is only 0.5 and $0.4 \mathrm{~K}$.

The free parameters in the model are the stellar masses $M_{1}$ and $M_{2}$, radii $R_{1}$ and $R_{2}$, temperatures $T_{1}$ and $T_{2}$, and six orbital parameters: inclination $i$, orbital period $P$, epoch of periastron $T_{p}$, argument of periastron $\omega$, eccentricity $e$, and systemic velocity $\gamma$ (held the same for both stars). The temperature is essentially unconstrained by the single-color broadband photometry, but the temperature ratio is weakly constrained by the ratios of radii and luminosities. While the models had freedom to vary the temperatures, the solution remained at the input spectroscopic temperatures. The individual RV measurements are fit, not just the $K_{1}$ and $K_{2} \mathrm{RV}$ amplitudes from the RV-only fit (i.e., we do not adopt the RV-only solution). The rotation of the stars, usually defined via the ratio $\Omega=\Omega_{\text {rot }} / \Omega_{\text {orb }}$, where $\Omega_{\text {rot }}$ is the stellar rotation angular frequency and $\Omega_{\text {orb }}$ is the orbital angular frequency, were also treated as free parameters. But for KOI-54 we defined these as $\Omega \equiv \Omega_{\mathrm{rot}} / \Omega_{\mathrm{ps}}$ where $\Omega_{\mathrm{ps}}$ is the "pseudosynchronous" rotation frequency and depends only on the eccentricity and orbital period as given by Equation (42) in Hut (1981):

$$
\Omega_{\mathrm{ps}}=(2 \pi / P) \times \frac{1+\frac{15}{2} e^{2}+\frac{45}{8} e^{4}+\frac{5}{16} e^{6}}{\left(1+3 e^{2}+\frac{3}{8} e^{4}\right)\left(1-e^{2}\right)^{3 / 2}}
$$

For an elliptical orbit true synchronous rotation is impossible, but there is a pseudosynchronous spin such that over the course of an orbit there is no net torque on the star's rotation, and so the spin will not evolve: $\dot{\Omega}=0$ (Hut 1981). Note that $\Omega_{\mathrm{ps}}$ is $\sim 20 \%$ slower than the orbital frequency at periastron, i.e., the spin is slower than what is necessary to keep the star tidally locked at periastron passage.

In addition, other "observed parameters" are used to constrain the model: $V_{\text {rot }} \sin i, \log g$, and the ratio of luminosities L2/L1. These are not fixed in ELC; rather, the models are steered toward them via a $\chi^{2}$ penalty. A genetic algorithm and Markov Chain Monte Carlo are used to find the best-fit models (in a $\chi^{2}$ sense) and confidence intervals. The best-fit model has a reduced $\chi^{2}$ less than 1 , indicating that the uncertainties in the light curve were overestimated in the data calibration; but we determined the $1 \sigma$ parameter intervals in the standard way by marginalizing over all other parameters and determining the interval bounded by $\chi_{\min }^{2}+1$; we did not decrease this interval to account for the reduced $\chi^{2}$ being less than 1 . 

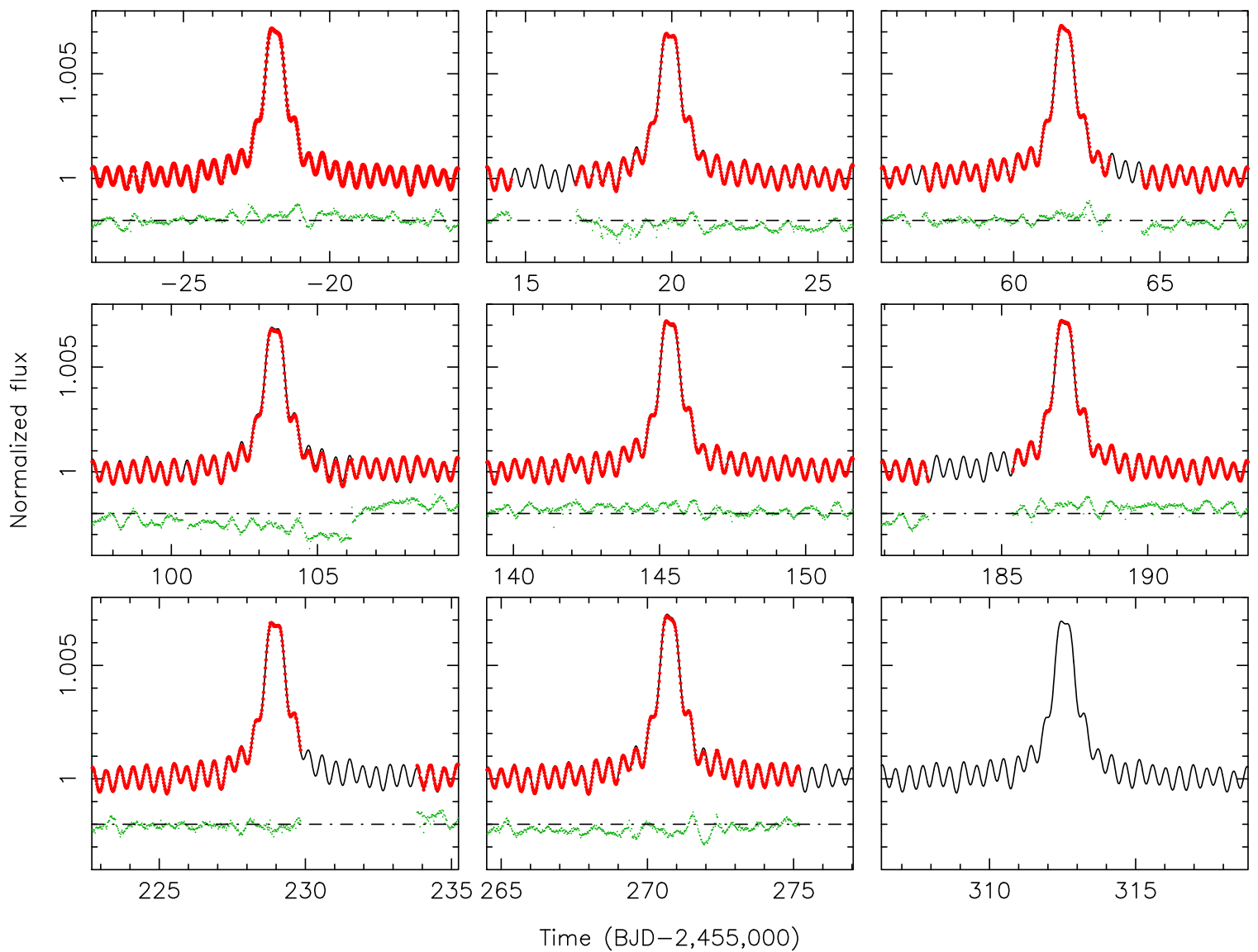

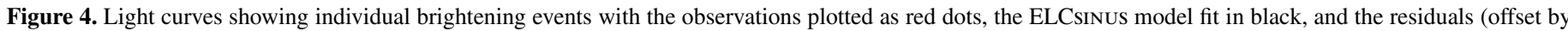
+0.998 and scaled by a factor of five) in green. The lower right-hand panel shows the model only.

(A color version of this figure is available in the online journal.)

\subsection{ELCSINUS}

The ELC code models the binary star light curve and $\mathrm{RV}$, but does not model the oscillations. Attempts to isolate the binary light curve from the pulsation light curve proved inadequate for the high precision Kepler observations, so we added the following functionality into the ELC code, now dubbed "ELCsinus." First, a trial binary star light curve is subtracted from the observed light curve. The mean is then subtracted and the Fourier transform is computed. The 15 largest peaks in the power spectrum are found (omitting sidelobes due to leakage of the two dominant peaks), and a sum of sines and cosines is made using the 15 measured amplitudes and phases. This 15 sine pulsation model is then added to the binary star model to create the light curve model. In this ELCsinus model, the pulsations modulate the average flux and are not scaled by the binary star light curve; thus they are not boosted during the brightening events. The radial velocities are unchanged. The ELCsinus model thus consists of a light curve, an RV curve for each star, and several derived parameters: L2/L1, and the $\log g$ and $V_{\text {rot }} \sin i$ for each star. All of these are used to compute the goodness-of-fit $\chi^{2}$ statistic and parameter uncertainty estimates.

As shown in Figure 4, we obtain an excellent fit to the light curve, matching the amplitude, shape, and phase of the brightening events. The brightening is due to a combination of mutual irradiation and tidal distortion during periastron passage of the pair of A stars on their highly eccentric, nearly face-on orbit. The system parameters of KOI-54 are reported in Table 2. The uncertainties listed are the formal uncertainties and should be treated as lower limits, as there are many potential sources of systematic errors as discussed in Section 5.5. As an example, the mass and radius of Star 1 from the ELCsinus fit yield a $\log g$ that is $1.6 \sigma$ larger than the spectroscopically measured $\log g$ (even though any deviation from the observed $\log g$ incurs a $\chi^{2}$ penalty). Although it is very difficult to assess the size of systematic uncertainties, a doubling of the formal uncertainties seems reasonable, especially for the inclination, masses, and radii of the stars.

Note that the star designated as Star 1 is the less massive star. Also note that the pulsations continue to occur during the brightening events and exhibit a fixed pattern: there is no discernable phase drift. The pulsations are matched well with our simple modification to ELC, indicating that the pulsations are not altered by the irradiation and gravitational distortions at periastron. Indeed, as discussed in the next section, the pulsations are driven by events at periastron passage.

\section{PERIASTRON-PUMPED PULSATIONS}

To investigate the pulsations, the best-fit binary star model was subtracted from the light curve. This leaves the stellar pulsations, random noise, systematic calibration noise, and any slight mismatches between the model and actual binary star light curve. The 15 sines used in ELCsinUs are not removed; only the binary star component of the model is removed.

The beat pattern seen in the light curve indicates that two closely spaced pulsations are present in the light curve. This is confirmed by the power spectrum of the pulsation-only light 

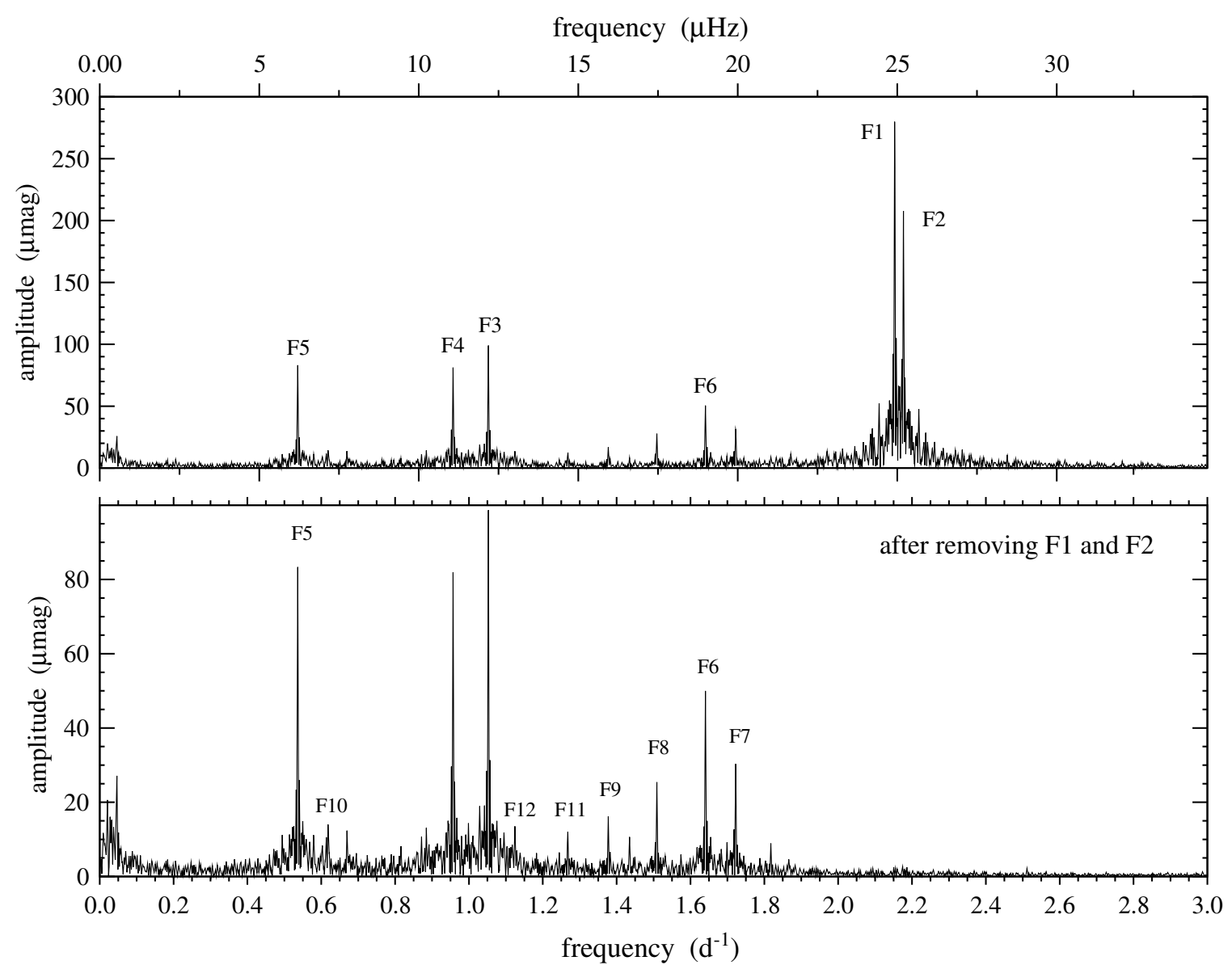

Figure 5. Power (amplitude) spectrum of the pulsation-only light curve, with the largest pulsations labeled. The bottom panel shows the power spectrum after prewhitening by removing the two dominant pulsations F1 and F2.

curve-see Figure 5. We computed the power spectrum using the PERIOD04 software package (Lenz \& Breger 2005), which uses an iterative least-squares fit to all detected sinusoidal terms simultaneously. Including the 2 dominant peaks in the power spectrum, there are 13 clean peaks with amplitudes greater than $12 \mu \mathrm{mag}$, corresponding to a signal-to-noise ratio $\gtrsim 20$. We list the 30 strongest pulsations in Table 3 . Frequencies less than $2 \mu \mathrm{Hz}$ (longer than $\sim 6$ days) are not included in the table as these long timescales suffer contamination from imperfect detrending, but we did include five low-frequency terms when computing the power spectrum. The largest spike, $\mathrm{F} 1$, is at a frequency of $2.15286 \mathrm{day}^{-1}(24.9174 \mu \mathrm{Hz})$ and the next largest, F2, at $2.17680 \mathrm{day}^{-1}(25.1944 \mu \mathrm{Hz})$, or roughly 11.15 and $11.03 \mathrm{hr}$. These pulsations are perfectly sinusoidal; there are no harmonics and no evidence of any modulation in frequency (caused, for example, by Doppler shifts due to orbital motion). We cannot tell which star is pulsating, or if both are, and if so, which pulsations originate on which star. The pulsation phases are equal very near (but not exactly at) the time of periastron, thus the beating envelope amplitude is largest near periastron and lowest at orbital phase 0.5. These two pulsations are also the fastest in the light curve; there is no significant power at frequencies above F2. More precisely, between $3 \mathrm{day}^{-1}(35 \mu \mathrm{Hz})$ and the Nyquist frequency for the LC data at $24.5 \mathrm{day}^{-1}(283 \mu \mathrm{Hz})$, there are no peaks above $3 \mu \mathrm{mag}$. The one month of SC data was also examined, after simply omitting the single brightening event during this month, and its power spectrum also reveals no signal greater than $3 \mu \mathrm{mag}$ out to 720 day $^{-1}(8.33 \mathrm{mHz})$, showing a complete absence of any $p$-mode pulsations. Thus, neither star is a $\delta$ Sct star to exquisitely high precision, which given their early A spectral type is somewhat surprising.

The separation between the two largest peaks, in period, is 41.771 days, very close to the orbital period of 41.805 days. Because the pulsations are not completely resolved in the power spectrum, their frequency difference is consistent with being identical to the orbital period. We assert that these are exactly the 90th and 91st multiples of the orbital frequency. This claim is confirmed by the other pulsations: 23 of the 30 largest pulsations are also very nearly exact integer multiples of the orbital frequency-see Table 3. Like the two dominant pulsations, these other pulsations are also very "pure," with no measurable deviations from being perfectly sinusoidal. (The naming of the pulsations roughly corresponds to the relative strengths of the pulsations, so F5 is the 5th largest pulsation. However, this is not an exact match because the amplitudes depend on the specific tapering method used, and as additional quarters of data were added, some spikes swapped relative heights.) We searched for additional patterns in the power spectrum other than harmonics of the orbital frequency. This led to numerous detections of frequency spacings within multiplets at a value of $\delta f \sim 0.132 \mathrm{day}^{-1}(\sim 1.53 \mu \mathrm{Hz})$, e.g., between F6 and F8, and F8 and F9-see Table 3. Given this strong coupling of the orbital frequency with the pulsation frequencies, it is very likely that the pulsations are a result of a resonance between the dynamic tides and one or more free low-frequency $g$-modes-see Aerts 
Table 3

Thirty Largest KOI-54 Pulsations

\begin{tabular}{|c|c|c|c|c|c|}
\hline ID & $\begin{array}{c}\text { Frequency } \\
\left(\text { day }^{-1}\right)\end{array}$ & $\begin{array}{c}\text { Frequency } \\
\quad(\mu \mathrm{Hz})\end{array}$ & $\begin{array}{l}\text { Amplitude } \\
\text { ( } \mu \mathrm{mag})\end{array}$ & $f / f_{\text {orbit }}$ & $\begin{array}{l}\text { Nearest } \\
\text { Harmonic }\end{array}$ \\
\hline $\mathrm{F} 1$ & 2.1529 & 24.917 & 297.7 & 90.00 & 90 \\
\hline $\mathrm{F} 2$ & 2.1768 & 25.195 & 229.4 & 91.00 & 91 \\
\hline F3 & 1.0525 & 12.182 & 97.2 & 44.00 & 44 \\
\hline $\mathrm{F} 4$ & 0.9568 & 11.074 & 82.9 & 40.00 & 40 \\
\hline F5 & 0.5363 & 6.207 & 82.9 & 22.42 & $\ldots$ \\
\hline F6 & 1.6405 & 18.988 & 49.3 & 68.58 & $\cdots$ \\
\hline F7 & 1.7222 & 19.933 & 30.2 & 72.00 & 72 \\
\hline F8 & 1.5087 & 17.462 & 17.3 & 63.07 & 63 \\
\hline F9 & 1.3773 & 15.941 & 15.9 & 57.58 & $\cdots$ \\
\hline F10 & 0.6697 & 7.751 & 14.6 & 28.00 & 28 \\
\hline F11 & 1.2678 & 14.673 & 13.6 & 53.00 & 53 \\
\hline F12 & 1.1241 & 13.011 & 13.4 & 46.99 & 47 \\
\hline F13 & 0.9329 & 10.798 & 12.5 & 39.00 & 39 \\
\hline F14 & 1.4349 & 16.608 & 11.6 & 59.99 & 60 \\
\hline F15 & 0.8851 & 10.244 & 11.5 & 37.00 & 37 \\
\hline F16 & 1.6983 & 19.656 & 11.4 & 71.00 & 71 \\
\hline F17 & 0.6183 & 7.156 & 11.1 & 25.85 & $\ldots$ \\
\hline F18 & 1.8178 & 21.039 & 9.8 & 75.99 & 76 \\
\hline F19 & 0.8574 & 9.924 & 9.3 & 35.84 & $\cdots$ \\
\hline F20 & 0.6458 & 7.475 & 9.1 & 27.00 & 27 \\
\hline F21 & 1.0284 & 11.903 & 8.4 & 42.99 & 43 \\
\hline F22 & 1.0765 & 12.460 & 8.3 & 45.01 & 45 \\
\hline F23 & 1.5092 & 17.467 & 8.1 & 63.09 & 63 \\
\hline F24 & 0.8610 & 9.965 & 6.9 & 35.99 & 36 \\
\hline F25 & 1.4452 & 16.726 & 6.8 & 60.42 & $\cdots$ \\
\hline F26 & 1.2439 & 14.397 & 6.4 & 52.00 & 52 \\
\hline F27 & 1.0078 & 11.664 & 6.3 & 42.13 & $\cdots$ \\
\hline F28 & 0.7894 & 9.137 & 5.9 & 33.00 & 33 \\
\hline F29 & 0.6937 & 8.028 & 5.8 & 29.00 & 29 \\
\hline F30 & 1.1483 & 13.290 & 5.7 & 48.00 & 48 \\
\hline
\end{tabular}

Notes. Uncertainty in frequencies is $\sim 0.0001 \mathrm{day}^{-1}$ or $0.001 \mu \mathrm{Hz}$. Formal uncertainty in amplitudes is $0.3 \mu$ mag. Orbital frequency $f_{\text {orbit }}$ was found via least-squares fit to best match the harmonics: $f_{\text {orbit }}=0.0239205 \mathrm{day}^{-1}=$ $0.276857 \mu \mathrm{Hz}$.

(2007) and Willems \& Aerts (2002) for a discussion of tidally driven pulsations in binary stars. We will return to discuss this in Section 5.3.

\section{DISCUSSION, CONCERNS, AND SPECULATION}

\subsection{Binary Star Phenomena}

KOI-54 is a remarkable binary star system. In the following section, we further discuss various properties of the system, but first we mention that if the Hipparcos parallax is reliable for the binary, the distance is $318 \pm 71 \mathrm{pc}$. At apastron the stars are separated by $0.79 \mathrm{AU}$, which translates to $2.5 \mathrm{mas}$ - a potentially resolvable separation.

\subsubsection{Photometric Determination of e and $i$}

The ELCsinus model produces an excellent fit to the photometry and tightly constrains the system parameters. To our knowledge, this is the first determination of stellar parameters for a non-eclipsing double-lined spectroscopic binary based on the brightening during periastron passage. This is possible because of (1) the high precision of the Kepler photometry and (2) the strong sensitivity of the brightening to periastron passage, and hence to the orbital parameters. Remarkably, we have found that the photometry alone can determine the orbital eccentricity as precisely as the radial velocities. This is a consequence of the dependence of the amplitude of the brightening to changes in eccentricity as illustrated in Figure 6. In this set of simulations, closely matched to KOI-54, the only parameter that varies is the eccentricity. In particular, the lower panel shows how one can estimate the eccentricity to $\sim 1 \%$ just from its amplitude, if all other parameters were known. The strong sensitivity to eccentricity is simply a consequence of the brightening being due to tidal forces and irradiation that scale as the separation of the stars cubed and squared, respectively, and the separation of the stars at periastron is linear in the eccentricity. (Note: we are assuming simple tidal distortion aligned along the line joining the center of masses; in the general asymmetric case the tidal force is much more sensitive to the separation, going as $F \propto r^{-7}$-see Hut 1981 for a discussion.)

In addition to the eccentricity, the Kepler photometry is also able to constrain the orbital inclination, even though there are no eclipses and no double-humped ellipsoidal variations are present. But in fact the ellipsoidal variations are present-for such a highly eccentric orbit the humps have shifted from the usual photometric quadrature phases, 0.25 and 0.75 , to the phase of periastron (defined here as phase 1.0), and the humps have merged. The two humps are not equal in height because the orbital inclination is not exactly zero and the orbital ellipses are not aligned along our line of sight (the argument of periastron is not $\pm 90^{\circ}$ ); thus the two phases of maximum visible ellipsoidal distortion are not symmetric about periastron. In addition, the inclination and argument of periastron determine the orientation of the irradiated hemispheres of the stars to our line of sight, and this creates a small asymmetry and shift in the phase of the brightening. For the geometry of KOI-54, the larger the inclination, the more the peak would shift to earlier orbital phases, and the narrower the peak the more asymmetric it would become (brighter post-peak than pre-peak). Such effects allow the inclination to be measured.

\subsubsection{Relative Contributions of Irradiation and Distortion}

Both tidal distortion and the irradiation/reflection contribute to the brightening, and Figure 7 shows the separate contributions of each. For KOI-54 the non-sphericity is the larger contributor, with the reflection component roughly $75 \%$ as large. The dominance of the ellipsoidal effect is not a necessity, but rather a consequence of the specific binary system parameters, and could reverse in another system (i.e., reflection effect $>$ ellipsoidal effect). As expected, the ellipsoidal component is more centrally peaked than the reflection component, as it is more sensitive to the separation of the stars. Note that the reflection component is slightly asymmetric, being brighter after periastron than before. This is a consequence of the inclination of the orbital plane and the orientation of the irradiated hemispheres with respect to the observer.

\subsubsection{Fit to the Radial Velocities}

While the ELCsinus model provides a good match to the Kepler photometry, the match to the radial velocities is not satisfactory at certain phases. The overall features are reproduced, and the rapid rise and fall of the velocities near periastron are well fitted, but the velocities at phases other than periastron are underestimated in a systematic way-see the residuals in Figure 3. When we fit the radial velocities without the photometry, we obtain much better agreement: reduced $\chi^{2}=1.00$ versus 1.61 for $(102-8=94)$ degrees of freedom-the solid and dashed RV curves in Figure 3 show the difference when using and not using the photometry. The maximum velocity 


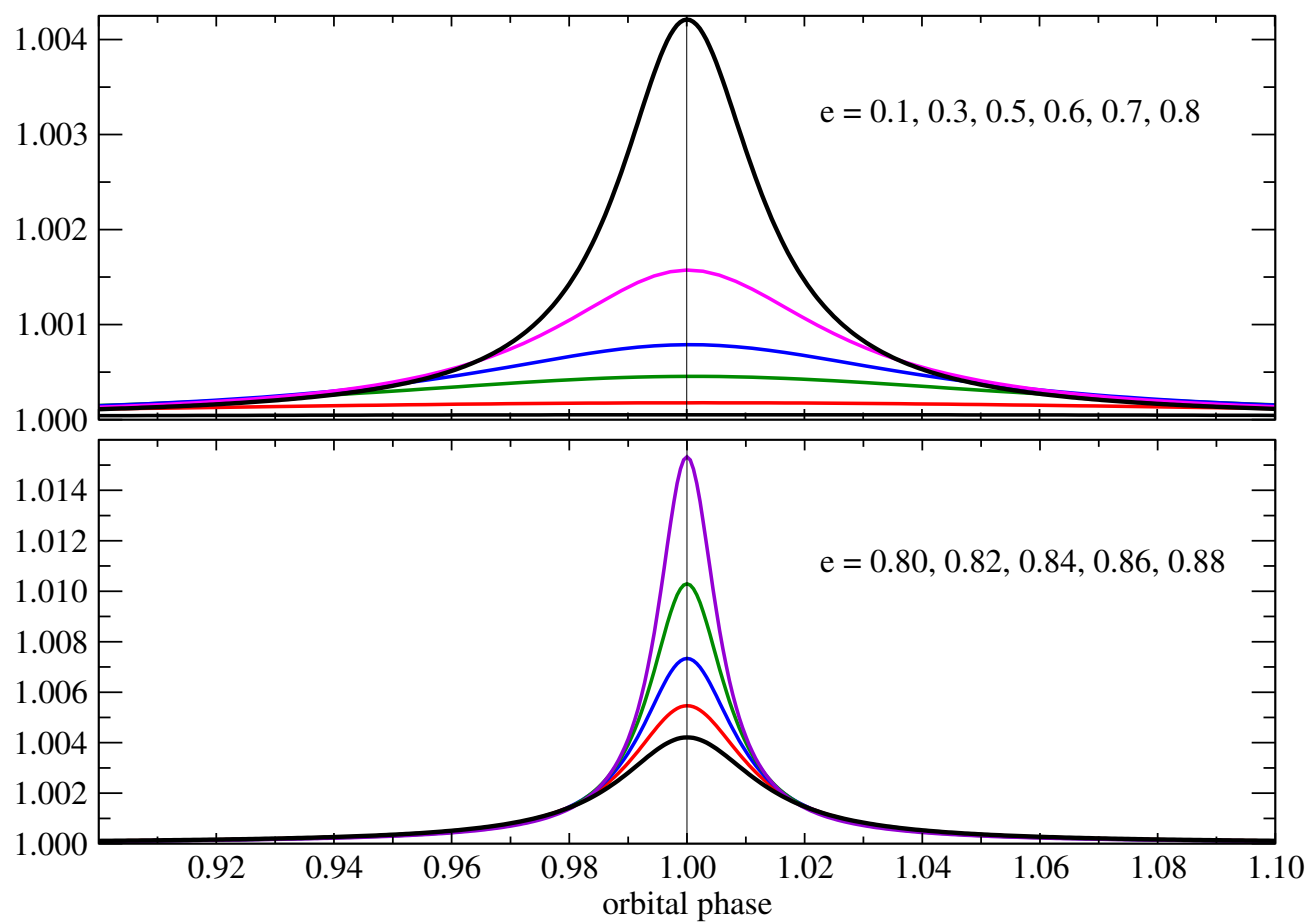

Figure 6. Illustrative ELC model light curves showing the effect of eccentricity on the amplitude of the brightening at periastron. Eccentricity increases from the lower curve to the upper curve. The strong sensitivity of the brightening to eccentricity allows the eccentricity to be determined independently of the radial velocities. The lower panel shows a tighter range of eccentricities, and by inspection, one can see that a brightening amplitude of $0.7 \%$ requires an eccentricity near 0.84 for this $i=5^{\circ}$ example.

(A color version of this figure is available in the online journal.)

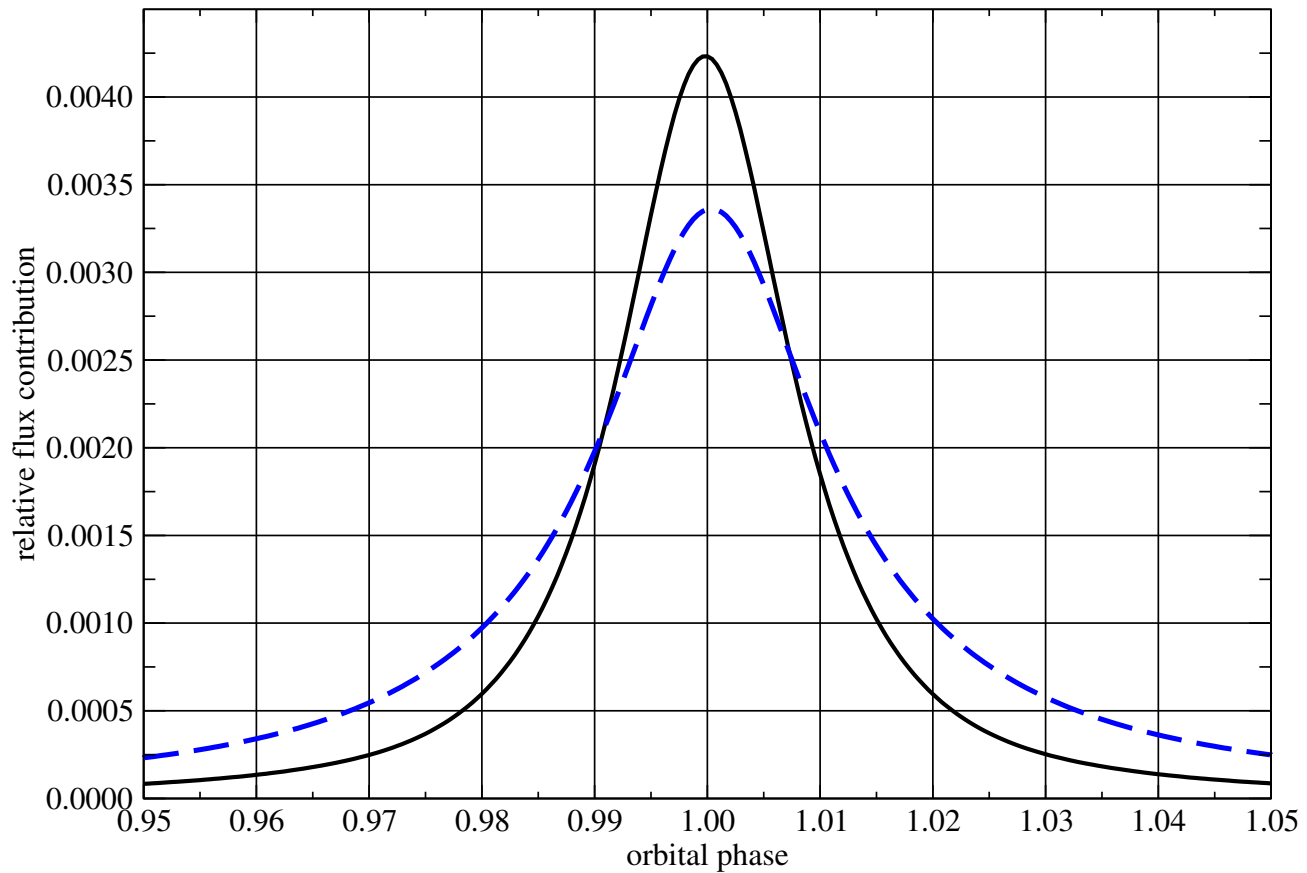

Figure 7. Relative contributions to the brightening from the tidal/ellipsoidal distortion only (solid curve) and irradiation/reflection only (dashed curve).

(A color version of this figure is available in the online journal.)

difference between the models is $0.3 \mathrm{~km} \mathrm{~s}^{-1}$ and occurs near orbital phase 0.1 , agreeing with where the deviation between the actual data and the ELCsinus fit are prominent. The fit is also poor near orbital phase 0.8 , and it is unclear if between these two phases the fit would show the same systematic underestimate, since most of this phase range lacks observations. As a check, we fit the velocities using a well-tested RV-only code and obtained the same results. Also note that ELC computes an intensity-weighted net RV, so that irradiation and tidal distortion are taken into account; e.g., center of light need not be the center of mass, especially at periastron. Thus, the best-fit solution using the photometry is not the best-fit solution for the radial velocities alone. To confirm this, we used the best-fit RV parameters to compute a light curve, and the result was a very poor 
match to the photometry. The origin of the photometric versus $\mathrm{RV}$ disagreement is not understood, and we can only speculate at this point. The poorest fit radial velocities are the ones with the lowest amplitudes, and these are the hardest to measure, as the lines from the two stars are most blended. This could account for most of the discrepancy. The stellar pulsations will affect the line profiles, thus adding jitter and broadening to the velocities which could induce a bias. The pulsations will also affect the measurement of $V_{\text {rot }} \sin i$, which could explain the wide range of $V_{\text {rot }} \sin i$ we measure, from $\sim 4$ to $10 \mathrm{~km} \mathrm{~s}^{-1}$. Finally, we have assumed that the stellar rotation axes are both aligned with the orbital axis. Although not expected to be misaligned (because the strong tidal forces will tend to align the spin axes and the measured $V_{\text {rot }} \sin i$ are the same for both stars), a misalignment could induce significant jitter into the radial velocities and bias in the $V_{\text {rot }} \sin i$ estimates.

\subsubsection{Tidal Evolution}

With its eccentricity of 0.83 , KOI-54 is very near the upper edge of the envelope of the distribution of eccentricities for its orbital period, $e=0.89$ following Mazeh (2008). The "periastron period," as introduced by Mazeh (2008), is the period of a circular binary having the same semimajor axis as the periastron distance, and for KOI-54 $P_{\text {peri -dist }}=2.8$ days. This short timescale suggests that the orbit should have experienced enough tidal evolution to synchronize over its main-sequence lifetime and possibly reduce its eccentricity from an even larger value. However, in his seminal work on the weak friction model for tidal interaction, Hut $(1981,1982)$ has shown that the evolution of eccentricity can be complex and depends strongly on the equilibrium ratio of orbital and rotational angular momentum, $\alpha$. In particular, for high values of $\alpha$, the evolution of eccentricity need not be monotonically decreasing, as the spin and orbital angular momentum can be exchanged back and forth. Furthermore, evolution of the stellar interior and its changing moment of inertia can alter simple orbital evolution (see Zahn 2008 for a discussion). While there is over a factor of 10 uncertainty in $\alpha$ due to dependence on the unknown internal mass distribution (parameterized by the radius of gyration) and the poorly known stellar rotation period, the ratio of orbit-tospin angular momentum is very large: $\sim$ hundreds to thousands. This is well over $\alpha_{\text {crit }}=35.447$ and tells us that the evolution of the orbit can be quite complex, with periods of increasing as well as decreasing eccentricity (Hut 1982).

Tidal forces can synchronize the stellar spin on a timescale 2-3 orders of magnitude faster than circularization (e.g., Zahn 2008), so despite the large eccentricity, we can expect the stellar rotation to have experienced significant evolution toward synchronicity with the orbit. The ratio of pseudosynchronous frequency to the orbital frequency is a function of the eccentricity only (Hut 1981; see Equation (1)), so we can compare this with the observations. The pseudosynchronous rotation frequency is $\Omega_{\mathrm{ps}}=2.48 \mathrm{day}^{-1}$, translating to a spin period of 2.53 days, $V_{\text {rot }}=43-47 \mathrm{~km} \mathrm{~s}^{-1}$, and a projected $V_{\text {rot }} \sin i=4.1-4.5 \mathrm{~km} \mathrm{~s}^{-1}$. This value roughly agrees with the lower range of our observed $V_{\text {rot }} \sin i$, and so a pseudosynchronous spin is roughly consistent with the observed $e, P$, and $V_{\text {rot }} \sin i$.

In our ELCsinus model we did let the stellar spins be free parameters; these are constrained mainly by the observed $V_{\text {rot }} \sin i$ but there is a weak dependence on the light curve because the oblateness of the stars depends on their rotation, and this affects the tidal distortion and reflection. In principle the stellar rotation can be refined through the light curve fit, and this could be used to check for pseudosynchronous rotation. But in practice this constraint is too weak to be useful. The models do have a marginal preference for Star 1 to have a rotation that is 3.5 times faster than pseudosynchronous, though the uncertainty admits the pseudosynchronous rate (there is a $\chi^{2}$ minima at $\Omega_{1} \sim 1$ ), and such a rotation rate yields a $V_{\text {rot }} \sin i$ that is $2 \sigma$ high compared to the observation. If Star 1 is indeed spinning faster than pseudosynchronous, it is unclear if the star is still spinning down but has not yet reached pseudosynchronism or if the star has exchanged angular momentum with the orbit and is being spun up from the pseudosynchronous rate. The observed slow rotation periods compared to normal A stars imply that both stars have experienced significant spin-down since birth; perhaps a spin-to-orbit exchange of angular momentum has prolonged or even increased the eccentricity of the orbit. However, Am and Ap stars may be born with a very slow spin, so this hypothetical exchange of spin and orbit angular momentum cannot be inferred.

\subsection{Exploratory Stellar Evolution Modeling}

We have begun a search of parameter space for coeval stellar evolution models that fit the stellar constraints as given in Table 2. We are using an updated version of the Iben $(1963,1965)$ stellar evolution code that includes the OPAL opacities (Iglesias \& Rogers 1996) supplemented by the Alexander \& Ferguson (1994) and D. Alexander (1995, private communication) low-temperature opacities and the Grevesse \& Noels (1993) solar element abundance mixture. As a starting point, we evolved an $M_{1}=2.33 M_{\odot}$ and an $M_{2}=2.40 M_{\odot}$ model with solar abundances: $X=0.68(\mathrm{H}$ mass fraction), $Y=0.28$ (He mass fraction), and $Z=0.04$ (mass fraction of elements heavier than $\mathrm{H}$ and $\mathrm{He}$ ). We find that the constraints in Table 2 are difficult to match with solarmetallicity $Z=0.02$ models, especially the combination of temperature and radius: the radii are too small for the temperatures. A metal abundance higher than $Z=0.04$ would allow a wider range of parameters of the evolution models to match the $\left(M_{1}\right.$, $\left.R_{1}, T_{1}\right)-\left(M_{2}, R_{2}, T_{2}\right)$ pair, and indeed the spectroscopy does indicate enhanced metallicity. However, for this initial investigation we did not further pursue the metallicity as this would also require varying the $Y$ abundance (beyond the scope of this investigation) and also that these stars may be metallic A stars (Am) so the surface abundances from spectroscopy may not be representative of the entire star.

In selecting models, we emphasized the constraints on the ratios of temperature, luminosity, and radius over their actual values as the ratios are better determined observationally. We discounted the spectroscopic log $g$ measurements that suffer from large uncertainties. To be specific, we sought to match the ratios in temperature $T_{2} / T_{1}=1.035 \pm 0.034$, luminosity $L_{2} / L_{1}=1.22 \pm 0.04$, and in radius $R_{2} / R_{1}=1.06 \pm 0.02$. As expected, a family of solutions was found; for example a pair of stars with temperatures $T_{1}, T_{2}$ of $8498 \mathrm{~K}, 8667 \mathrm{~K}$ satisfies our matching criteria, as does another pair at $8754 \mathrm{~K}, 8999$ $\mathrm{K}$. Looking more closely, in the cooler pair, with an age of $0.354 \mathrm{Gyr}$, the temperature, luminosity, and radius ratios (1.020, 1.26, and 1.08) are close to the measured values, but the absolute radii $\left(R_{2}=2.52\right.$ and $\left.R_{1}=2.35 R_{\odot}\right)$ are somewhat larger than our estimated values of 2.33 and $2.20 R_{\odot}$. In the hotter pair, with an age of $0.263 \mathrm{Gyr}$, the temperature, luminosity, and radius ratios $(1.028,1.25$, and 1.06) are again close to the measured values, and the absolute radii ( 2.28 and $2.15 R_{\odot}$ ) are closer to the reported values, but the temperatures are at the high end of 
the uncertainty range. From this exploratory investigation, the important conclusion we can draw is that the $\left(M_{1}, R_{1}, T_{1}\right)-\left(M_{2}\right.$, $\left.R_{2}, T_{2}\right)$ set we measure is consistent with stellar evolution models of identical age and metallicity stars.

\subsection{Tidal Pulsation Modeling}

By way of brief introduction, linear perturbation theory can be used to determine the pulsation frequencies in stars, given their internal structure. Stellar pulsations are generally of two classes, the $p$-modes or $g$-modes depending upon whether pressure or gravity is the local restoring force. Being acoustic waves, $p$-mode characteristics are dependent on the local sound speed, and are important in the envelope and outer portion of the star. Their motion is primarily vertical and they have periods of the order of hours and less. The $g$-modes are set up by the competition between gravity and buoyancy deep within the star. Their wave motion is primarily horizontal and they have periods of the order of one day (for non-degenerate stars). The $g$ modes cannot extend through convective zones as the convective instability means there is no local restoring force. The modes are classified according to three spherical harmonic integers: the number of radial nodes $(n)$, the angular degree $(\ell)$, and azimuthal order $(m)$. ("Nodes" are the locations where the standing wave pattern, caused by the interference of the pulsations, has zero amplitude.) The degree $\ell$ gives the number of nodal circles on the sphere, and $|m|$ is the number of nodal lines that cross the equator (or equivalently, go through the poles). Radial modes do not change the spherical symmetry of the star during the pulsation cycle and are characterized by $\ell=m=0$. The frequencies of non-radial modes of degree $\ell$ are split by rotation or magnetic fields. Rotation lifts the degeneracy in $m$ in such a way that each mode of degree $\ell$ results in $2 \ell+1$ multiplet components in the frequency spectrum, with $m=-\ell, \ldots, 0, \ldots,+\ell$. The classical $\kappa$ (opacity valving) mechanism caused by He ionization in the stellar envelopes is predicted to drive $p$-mode pulsations in A- to early F-type main-sequence stars, while $g$-mode pulsations are not expected in these types of stars. For a complete reference see Aerts et al. (2010).

The periodic tidal forces experienced by the stars at periastron passage are expected to induce stellar oscillations, including $g$-modes which otherwise would not be present. To explore such tidal excitation, we assume one of the stars $\left(M_{1}\right)$ to rotate rigidly and the other star $\left(M_{2}\right)$ to be well described as a point source. Whenever the ratio of the external tidal force to the self-gravity at the star's equator is small, i.e., the dimensionless parameter $\varepsilon_{\mathrm{T}} \equiv\left(R_{1} / a\right)^{3} M_{2} / M_{1} \ll 1$, the tide-generating potential in the eccentric orbit can be expanded as a series in terms of spherical harmonics and of the companion's mean motion. We follow the method outlined in detail in Willems \& Aerts (2002), who have shown that the spherical harmonic with degree $\ell=2$ is by far the dominant term in the expansion of the tide-generating potential, and only components with $m=-2,0,2$ occur. The values of these components depend on the orbital inclination and eccentricity, as well as on the mean and true anomaly of the companion in its relative orbit. Whenever the forcing frequency, $\sigma_{\mathrm{T}}=k \Omega_{\text {orb }}+m \Omega_{\text {rot }}$, matches the star's free gravity oscillation modes, the tidal action exerted by the companion gets enhanced and the particular oscillation mode gets resonantly excited with the forcing frequency of the dynamic tide (Smeyers et al. 1998). Thus, we calculated the $\ell=2, m=0 \mathrm{~g}$-mode eigensolutions for each of these stars using the procedure and non-adiabatic nonradial pulsation code of Pesnell (1990) as reported in Guzik et al. (2000). We find that the $g$-mode spectrum for stars of this type is very dense, with period spacings of the order of 0.03 days. These are damped oscillations that would die out without the periodic tidal "pumping," but the damping times are very much longer than the orbital period (on the order of 1000 years), so once a mode is excited, it is certainly not expected to change amplitude during an orbital period-or the lifetime of the Kepler Mission.

While still adhering to the constraints on the stars discussed above, we tweaked the tidal model parameters to find a star whose $\ell=2, m=0$ spectrum has one mode that coincides exactly with F1 (i.e., 90 times the orbital frequency). This model has a mass of $2.33 M_{\odot}, T_{\text {eff }}=8663 \mathrm{~K}$, and $R=2.15 R_{\odot}$, and most importantly, its $g_{14}$ mode of $\ell=2, m=0$ has frequency $\mathrm{F} 1$, proving that standard stellar models exist that can generate a resonant tidal excitation. (The $g_{14}$ mode has $n=14$ radial nodes in the interior of the star and the $\ell=2, m=0$ is a quadrupole that distorts the star in the correct directions in response to tidal forcing.) Even though we did not tune the predictions of the tidal excitation theory to fit for KOI-54 in this discovery paper, we expect a frequency pattern as in the example treated in Willems $\&$ Aerts (2002), i.e., a pattern of resonances at spaced values of the rotational and orbital frequency as $k \Omega_{\text {orb }}+m \Omega_{\text {rot }}$. We applied this to all the frequencies detected in KOI-54 and their splitting values, which allowed us to deduce that $\Omega_{\text {rot }} \sim 0.133$ day $^{-1}$ $(1.54 \mu \mathrm{Hz})$ from the occurrence of $m=1$ and $m=2$ tidal splittings. This translates into a mean rotation period of 7.5 days and an equatorial rotation velocity of some $15 \mathrm{~km} \mathrm{~s}^{-1}$. If the orbital and rotational axes are aligned, as assumed in the tidal theory, this leads to a prediction of $V_{\text {rot }} \sin i$ of $1.5 \mathrm{~km} \mathrm{~s}^{-1}$. This rotation velocity is certainly low compared to the observed $V_{\text {rot }} \sin i$, being $\sim 1.7 \sigma$ from our adopted value, but we remark that the $V_{\text {rot }} \sin i$ measurements were made in the standard way and did not take into account pulsational broadening of the lines. We therefore tentatively accept the size of the tidal splitting of the lines as compatible with the measured $V_{\text {rot }} \sin i$. Furthermore, it is unlikely that the rotation inside the star will be rigid, and so this value of $\Omega_{\text {rot }}$ should be interpreted as an average value over the depth of the star, effectively negating concerns that this average rotation velocity is too low compared to the observed surface $V_{\text {rot }} \sin i$. In summary, we have found a model consistent with the observations that can explain all but one of the 30 largest observed pulsations as either harmonics of the orbital frequency or $m=1$ and $m=2$ tidal splittings of those harmonics. (F25 remains unaccounted for in this model.) Despite the very attractive features of this model, it is still very exploratory in nature and we do not claim uniqueness of the solution. Future observations will lead to much better frequency resolution, allowing a much more refined stellar model. We point out that slight deviations of the observed frequencies of the splittings from their exact predicted frequencies is quite interesting, and probably related to the nonrigidity of the stellar rotation. When several years of Kepler observations are available, we have the exciting prospect of using the observed tidal splittings to allow for an asteroseismic determination of the internal rotation law of the star.

\subsection{Pulsations Puzzles}

From their spectroscopic temperatures $\left(T_{\text {eff }} \sim 8500,8800 \mathrm{~K}\right)$ both stars are too hot to be $\gamma$ Dor pulsators, and the absence of any periodicities shorter than $11 \mathrm{hr}$ in the Short Cadence light curve power spectrum confirms this. Why the stars do not exhibit any $\delta$ Scuti-like pulsations is not known, though it might be that the stars are just beyond the blue edge of the classical instability 
strip. Presumably no unstable modes ( $p$ or $g$ ) exist for the stars, and the stars are intrinsically non-pulsators. This supposition is supported by the exploratory stellar models discussed in the previous section.

The pulsations that are observed are those modes for which their free $g$-mode eigenfrequency is close enough to a harmonic of the orbital period that they are resonantly driven to measurable amplitudes by the periodic tidal (and possibly thermal) forces felt at periastron. Thus while very many pulsation modes are possible, only those that are integer multiples of the orbital frequency and phase-locked with the binary orbit are present.

Some challenges posed by the pulsations in KOI-54 include: (1) Why are the 90th and 91st harmonics of the orbital frequency the strongest pulsations? (2) Why should one particular mode be strongly excited while many adjacent ones are not, i.e., why is orbital harmonic 91 very strong but 92 completely absent? (3) What limits the highest frequency excited mode and why is there no significant power present at higher harmonics? (4) Why are some modes tidally split but not others? and (5) If only one of the stars is pulsating, which one is it, and why is the other not pulsating?

Interestingly, if we accept the slow stellar rotation inferred from the tidal splitting of the pulsations, that would mean the star is rotating at only a third of the pseudosynchronous rate. This would seem unlikely in a simple tidal evolution scenario, but the very large value of orbit-to-spin angular momentum ratio means that exchanges of angular momentum are possible and episodes of spin-up and spin-down can take place. Or, as mentioned earlier, the stars are possibly Ap stars that may have been born rotating very slowly. A factor-of-two improvement in the measured $V_{\text {rot }} \sin i$ would be very valuable; the precision is currently available, but the accuracy is not.

Given that the stars are similar in mass, radius, and temperature, have identical ages and metallicities, and experience identical driving frequencies, a total lack of pulsations from one of the stars would be puzzling. And yet the matching of the pulsation frequencies with a single pulsating star, and the lack of any apparent double set of peaks in the power spectrum, does suggest that one star could be responsible for all the pulsations. In principle, high spectral resolution, time-resolved spectroscopy can determine which of the stars is pulsating (or if both are). If the spectra are obtained near periastron, the component lines will be well separated and pulsations in one of the sets of lines immediately tells us which star is pulsating. However, the intensity amplitude of the pulsations is very small, $\sim 0.1 \%$, making this rather challenging. Velocity modulations causing line profile shape changes are much more likely to be observable, though again the expected variations will be small. Further complicating the matter is the need to cover at least one full oscillation cycle, thus requiring $>12 \mathrm{hr}$, and perhaps a multi-longitude campaign. Fortunately, the star is bright $(\mathrm{Kp}=8.380)$ and the integrations can be long and still temporally resolve the pulsations. The reward for such efforts is high: given that the two stars are very similar, if only one star is pulsating, it means that the excitation mechanism must be very fine-tuned to drive the oscillations in one star but not the other. Thus, there is the potential for a precision investigation of internal stellar structure, given stars of identical age and metallicity and nearly identical masses.

\subsection{Additional Systematic Concerns}

While we are confident that the periastron-pumped pulsating binary star model is correct, given the complexity of both the physics and the data calibration, several sources of systematic error are potentially present. In this section, we describe several of these, with an assessment of their effects when possible. These are checks of the robustness of the overall solution, not detailed investigations that could reveal small changes at the few- $\sigma$ level. Toward that goal, we used a linear limb-darkened blackbody approximation instead of stellar atmospheres and locked the stellar rotation to the pseudosynchronous spin frequency. We begin with a look at the data calibration.

The most significant of the systematic errors in the data arise from the difficulty in removing small and abrupt changes in the flux level due to changes in pointing, cosmic ray events, safe modes, etc. While such breaks are usually visible in Kepler light curves, the pulsations in KOI-54 hide these. The residuals of the ELCsINUs fit exhibit small but significant meanderings and tilts on longish timescales (days to weeks) which are almost certainly due to detrending problems. Figure 4 shows the residuals of the data minus the ELCsINUs fit scaled by a factor of five to be more visible. The residuals are not white noise, indicating correlations due to the detrending, but also due to the fact that the model includes only 15 sinusoids while at least 30 are present. The largest set of correlated residuals occurs at the brightening event near day BJD 2,455,105 (see the middle left panel in Figure 4). Because the determination of the eccentricity and inclination is so sensitive to the amplitude and shape of the brightenings, even small tilts or jumps could potentially affect these estimates. We were concerned about the inclination in particular. Normally (e.g., for eclipsing binaries) a small change in inclination does not significantly affect the mass estimates for the stars, as the slope of the sine function near $90^{\circ}$ is flat. But for KOI-54 the inclination is small so changes in $\sin i$ go linearly with $i$. Because the masses depend on $\sin ^{-3} i$ a change from $5^{\circ}$ to $6^{\circ}$ results in a decrease of $58 \%$ in mass. To see if detrending imperfections could affect the accuracy of our model parameters (as opposed to precision), we carried out two investigations. First, we masked out the region with the largest residuals and re-fitted. Second, we used the power spectrum feature in ELCsInUs to measure, then remove, low-frequency power in the residuals, on timescales of 10 days and longer. While the $\chi^{2}$ of the models was significantly better (by construction), there was no significant change in the system parameters.

The determination of the eccentricity depends on the relative flux increase of the brightening event. Thus any systematic error in normalization will affect the eccentricity. The light curve is in fact contaminated by background starlight contained in the same aperture used for KOI-54, and the relative amplitude of the brightening is thus biased low; hence the derived eccentricity is potentially biased low. To check the effect this may have, we subtracted $250 \mathrm{ppm}$ from the light curves and re-fitted. In addition, we included a small "third light" dilution factor into the model and fitted for this parameter. In both cases, there was no significant change in the model parameters. Furthermore, such a bias would not effect the RV-only eccentricity estimate, which agrees remarkably well with the photometric estimate.

The "wings" of the brightening event (i.e., the upward curvature of the light curve) extend all the way to apastron at phase 0.5 ; there is no phase where the light curve is truly flat. This is a potential problem in the calibration: we detrended the light curve assuming it was flat between phases 0.1 and 0.9 . From our binary-star only light curve, the maximum difference in relative flux between phases 0.1 through 0.9 is $99 \mathrm{ppm}$, roughly 1.7 times the adopted uncertainty per point. However 
the light curve is flat to within $25 \mathrm{ppm}$ from phases $0.2-0.8$, so the neglect of curvature in the calibration is benign.

In our model, we have neglected several physical effects which we address below. Systematic errors in the model are potentially more serious than those of data calibration, as we have eight brightening events that can help average over any calibration errors. For example, as is standard practice, we have ignored any consideration of magnetic fields. However, for these Am or Ap stars, such neglect at periastron passage may not be entirely justified and warrants further investigation.

We have not included relativistic Dopper boosting of the light curves. For this nearly face-on binary this boosting effect will be very small, and a quick test showed that including the boosting produced a change in total (not reduced) $\chi^{2}$ of $\sim 0.1$, a completely negligible amount. Nevertheless, in general, future models should include this effect as a modestly higher orbital inclination would result in a measurable difference in the light curve, given Kepler's remarkable precision.

In general, an irradiated star is hotter than an un-irradiated star, and so the light that impinges back onto the irradiating star is greater than if the irradiated star were isolated. Thus the reflection effect should be iteratively computed, and this is the standard method championed by Wilson (1990). However, for KOI-54 this is a very small effect as the total brightening is less than $1 \%$, and tests with iteration showed almost no difference compared to a single calculation assuming a pointsource irradiator (which is exact for perfectly spherical stars). Given the large computational cost of full geometry tile-by-tile iteration and the negligible benefit, we used the point-source irradiation approximation. Our models have a maximum nonsphericity of $0.7 \%$ at periastron (ratio of minimum-to-maximum radii: pole radius to L1-direction radius), justifying the spherical approximation for irradiation. And despite the relatively close periastron passage of $0.065 \mathrm{AU}$ ( $\sim 6.4$ stellar radii), the stars only fill $\lesssim 30 \%$ of their Roche lobe radii.

The most significant of the limitations of our model is the use of the Roche approximation. While quite successful at matching the observations, there is a systematic bias inherent in the use of Roche potentials in that the tidal elongations are always pointing along the axis connecting the centers of mass. In other words, the stars are treated as perfect, viscousless fluids that can instantaneously adjust their shapes to the gravitational and spin potentials. ${ }^{22}$ But real stars will have tidal bulges that do not instantly readjust to the changing external potential and are also "dragged" by the rotation of the star. Thus, the elongation will not be aligned with the center of masses except when the stars are in synchronous rotation in a circular orbit. The irradiation/reflection would also be affected, simply because of the different geometry. Furthermore, the Roche potential treats the mass distribution as a point-mass at the center of the star; this is equivalent to an $n=5$ polytrope or a tidal Love number $k_{2}=0$. Thus for a given external force, the Roche potential yields the least possible tidal deformation. It then follows that the amplitude of the ellipsoidal variation is minimal in the Roche approximation. While it is true that the mass inside stars is highly centrally concentrated, a more realistic mass distribution would produce a larger tide for a given external force. So to match a given observed brightening amplitude, a weaker tidal force would be required. This could be achieved by either lower masses, or much more likely, a very small increase in separation

\footnotetext{
22 The Roche potential approximation is similar to the simplest equilibrium
} tide models in this regard. at periastron due to a very small decrease in eccentricity. Hence the brightening in the Roche potential model is expected to be very slightly different than a case where a more realistic internal mass distribution is used.

Although we have measured very precise system parameters with our ELCsInUs model, for the reasons discussed above the accuracy is worse than the precision. This is especially true for the eccentricity and inclination, and therefore the stellar masses which are proportional to the cube of the inclination. On the other hand, the photometric-only eccentricity agrees well with the RV-only eccentricity, indicating that the Roche approximation is valid at the level of analysis presented in this discovery investigation of KOI-54. A better treatment of the system, well beyond the scope of this paper, would involve dynamical tide theory - particularly relevant given the observed tidally driven pulsations.

This deficiency of the Roche model leads to an interesting supposition. The angle between the instantaneous centers of the stars and the axis of the tidal bulge depends on the internal structure of the star and is related to the efficiency of tidal dissipation. Measurement of this lag angle can then be used to constrain the tidal quality factor $Q$. If one could determine the instantaneous geometry of the system without the photometry, then the lag between the brightening and the true time of periastron passage could be measured. The geometry could in principle be determined though a great host of radial velocities, corrected for the distortions caused by the pulsations. Another possible way is related to the pulsations themselves; they provide a clock that could be used to set a fiducial time against which to measure the time of brightening. Interestingly, the time when the two largest modes (F1 and F2) are in phase is not the time of periastron as defined by the photometry, but $0.39 \pm 0.07$ days $(=9.4 \mathrm{hr})$ earlier. This can actually be seen in the light curves themselves, as the asymmetric shoulders of the brightening and dip at the very pinnacle-see Figures 1 and 4 .

\subsection{KOI-54 in Context}

While tidally excited oscillations have been studied in quite some detail from a theoretical point of view (e.g., see Aerts et al. 2010 for a discussion and references), observational evidence of their existence is scarce. Prior to KOI-54, only three such systems were known to exist. The rarity of these stars is understandable if one realizes that the forcing frequencies from the dynamical tides must come very close to the free eigenmode frequencies of one of the stars in order to resonantly excite the oscillation (e.g., Willems 2003). This naturally leads to the excitation of particular gravity modes whose frequencies are exact multiples of the orbital frequency.

Two of the previously known cases, HD 177863 (De Cat et al. 2000) and HD 209295 (Handler et al. 2002), were discovered from ground-based photometry and spectroscopy and are single-line spectroscopic binaries. HD 177863 is a slowly pulsating B star with $e=0.60, P_{\mathrm{orb}}=11.9$ days with one detected gravity mode whose frequency is 10 times the orbital frequency. HD 209295 is a hybrid $\gamma$ Dor/ $\delta$ Sct star in a binary with $e=0.35, P_{\mathrm{orb}}=3.1$ days with five $g$-modes having frequencies which are exact multiples of the orbital frequency, besides a free $\delta$ Sct mode. A much more interesting case is the eclipsing double-lined spectroscopic B-type binary HD 174884 discovered with the CoRoT mission (Maceroni et al. 2009). This system has $e=0.29, P_{\text {orb }}=3.66$ days and has pulsations with exact multiples of $2,3,4,8$, and 13 times the orbital frequency and very tightly constrained physical parameters. 
While it is unclear if the lowest-order multiples are due to imperfect prewhitening of the orbital curve (i.e., removal of the binary star contribution to the light curve) one does not expect this for harmonics 8 and 13 as all lower-order harmonics should have been found as well. Moreover, these two higher frequencies correspond exactly to those of free gravity modes of radial order $\sim 10$, which points to tidal excitation.

With its periodic tidal brightening and rich set of pulsations (over 30 pulsations at either integer multiples of the orbital frequency or at tidally split multiples of the orbital frequency are present), KOI-54 now joins this elite set of eccentric binaries that exhibit tidally driven pulsations.

\section{SUMMARY}

Far from being an ordinary A star, the exquisite Kepler observations of KOI-54 have revealed the object to be a fascinating binary star system exhibiting a host of interesting phenomena. We have successfully matched the light curve and radial velocities with a model consisting of a pair of $\mathrm{A}$ stars on a highly eccentric $(e=0.83)$ orbit, seen nearly faceon $(i=5.5)$. As the stars closely pass each other at periastron, coming within $\sim 6$ stellar radii, the stars tidally distort each other's shape and mutually irradiate and heat each other. The combination of the tidal ellipsoidal variation and the irradiation/reflection effect creates the periodic $0.7 \%$ brightening seen every 41.8 days. In addition, the close periastron passage is responsible for exciting a rich set of stellar pulsations with at least 30 modes. The two largest pulsations, at the 90th and 91st harmonics of the orbital frequency, beat against each other producing the modulation envelope seen in the light curve. The remaining pulsations are explained as additional harmonics of the orbital frequency or tidally split harmonics of those frequencies, clearly establishing the tidally driven origin of the pulsations. Using these frequencies we are able to deduce the average rotation period of the pulsating star, 7.5 days. KOI-54 is the only case where the tidally excited oscillations have allowed such a measurement. Future Kepler observations will allow factors of several times higher precision frequency measurements, offering the potential to map the internal rotation profile of one of the KOI-54 stellar components.

Despite our success in modeling the system, many puzzles remain. For example, it is not known which of the stars is pulsating, or if both are. Given that the stars are similar in mass and radius, and identical in age and metallicity, it is not known why one star would pulsate and the other not. Finally, although the pulsations and brightening events appear quite dramatic in the light curve, the amplitude of the pulsations is in fact considerably less than $0.1 \%$; the discovery of the nature of this remarkable star system was made possible by the extraordinary precision and duration of the Kepler photometry.

Kepler was selected as the 10th mission of the Discovery Program. Funding for this mission is provided by NASA, Science Mission Directorate. The authors acknowledge support from the Kepler Participating Scientists Program via NASA grant NNX08AR14G. C.A. and W.Z. received funding from the European Research Council under the European Community's Seventh Framework Programme (FP7/2007-2013)/ERC grant agreement No. 227224 (PROSPERITY). Some of the data presented herein were obtained at the W. M. Keck Observatory, which is operated as a scientific partnership among the California Institute of Technology, the University of California, and the National Aeronautics and Space Administration. The Observatory was made possible by the generous financial support of the W. M. Keck Foundation. We thank Gur Windmiller for general assistance and for a careful reading of this manuscript. We especially thank the many members of the Kepler team whose hard work made these observation possible. Finally, we thank the anonymous referee for a thorough review of this paper.

Facilities: Kepler, Keck:I (HIRES), FLWO:1.5m (TRES), HET (HRS), Shane (Hamilton Echelle Spectrograph), Smith (Coude Spectrograph), NOT (FIES)

\section{REFERENCES}

Aerts, C. 2007, in IAU Symp. 240, Binary Stars as Critical Tools \& Tests in Contemporary Astrophysics, ed. W. I. Hartkopf, E. F. Guinan, \& P. Harmanec (Cambridge: Cambridge Univ. Press), 432

Aerts, C., Christensen-Dalsgaard, J., \& Kurtz, D. W. (ed.) 2010, Asteroseismology (Heidelberg: Springer)

Alexander, D., \& Ferguson, J. W. 1994, ApJ, 437, 879

Avni, Y., \& Bahcall, J. N. 1975, ApJ, 197, 675

Borucki, W. J., Koch, D., Basri, G., et al. 2010, Science, 327, 977

Claret, A. 2000, A\&A, 359, 289

De Cat, P., Aerts, C., De Ridder, J., et al. 2000, A\&A, 355, 1015

Gilliland, R. L., Jenkins, J. M., Borucki, W. J., et al. 2010, ApJ, 713, L160

Grevesse, N., \& Noels, A. 1993, in Origin and Evolution of the Elements (Cambridge: Cambridge Univ. Press), 15

Guzik, J. A., Kaye, A. B., Bradley, P. A., Cox, A. N., \& Neuforge, C. 2000, ApJ, 542, L57

Handler, G., Balona, L. A., Shobbrook, R. R., et al. 2002, MNRAS, 333, 262

Hauschildt, P. H., Baron, E., \& Allard, F. 1997, ApJ, 483, 390

Hut, P. 1981, A\&A, 99, 126

Hut, P. 1982, A\&A, 110, 37

Iben, I. 1963, ApJ, 138, 452

Iben, I. 1965, ApJ, 141, 993

Iglesias, C., \& Rogers, F. J. 1996, ApJ, 484, 943

Jenkins, J. M., Caldwell, D. A., Chandrasekaran, H., et al. 2010a, ApJ, 713, L87

Jenkins, J. M., Caldwell, D. A., Chandrasekaran, H., et al. 2010b, ApJ, 713, L120

Kallrath, J., \& Milone, E. F. 1999, Eclipsing Binary Stars (New York, NY: Springer)

Koch, D. G., Borucki, W. J., Basri, G., et al. 2010, ApJ, 713, L79

Lenz, P., \& Breger, M. 2005, Commun. Asteroseismol., 146, 53

Maceroni, C., Montalbán, J., Michel, E., et al. 2009, A\&A, 508, 1375

Machalek, P., \& Christiansen, J. 2010, Kepler Data Release 8 Notes KSCI19048-001 NASA Ames Research Center, Moffett Field, CA, available at http://archive.stsci.edu/kepler/data_release.html

Mazeh, T. 2008, in Tidal Effects in Stars, Planets and Disks, ed. M.-J. Goupil \& J.-P. Zahn (EAS Publications Series, Vol. 29; Paris: EDP Sciences), 1

Orosz, J. A., \& Hauschildt, P. H. 2000, A\&A, 364, 265

Pesnell, W. D. 1990, ApJ, 363, 227P

Sahu, K. S., \& Gilliland, R. L. 2003, ApJ, 584, 1042

Sepinsky, J. F., Willems, B., \& Kalogera, V. 2007, ApJ, 660, 1624

Smeyers, P., Willems, B., \& Van Hoolst, T. 1998, A\&A, 335, 622

Sneden, C. 1973, ApJ, 184, 839

Van Cleve, J., \& Caldwell, D. 2009, Kepler Instrument Handbook KSCI-10933-001, NASA Ames Research Center, Moffett Field, CA, available at http://keplergo.arc.nasa.gov/Instrumentation.shtml, and also http://keplergo.arc.nasa.gov/calibration/KSCI-19033-001.pdf

von Zeipel, H. 1924, MNRAS, 84, 702

Willems, B. 2003, MNRAS, 346, 968

Willems, B., \& Aerts, C. 2002, A\&A, 384, 441

Wilson, R. E. 1990, ApJ, 356, 613

Zahn, J.-P. 2008, in Tidal Effects in Stars, Planets and Disks, ed. M.-J. Goupil \& J.-P. Zahn (EAS Publications Series, Vol. 29; Paris: EDP Sciences), 67 Zucker, S., \& Mazeh, T. 1994, ApJ, 420, 806 